\title{
Conscious Access In The Near Absence Of Attention: Critical Extensions On The Dual-task Paradigm
}

\author{
Julian Matthews ${ }^{1,2}$, Pia Schröder², Lisandro Kaunitz ${ }^{2}$, \\ Jeroen van Boxtel ${ }^{2,3}$, Naotsugu Tsuchiya ${ }^{2,3}$ \\ ${ }^{1}$ Cognition and Philosophy Lab, Faculty of Arts, Monash University, Clayton 3800, Victoria, Australia. \\ ${ }^{2}$ School of Psychological Sciences, Monash University, Clayton 3800, Victoria, Australia. \\ ${ }^{3}$ Monash Institute of Cognitive and Clinical Neurosciences, Monash University, Clayton 3800, Victoria, Australia.
}

Accepted October 20th, 2017.

An abridged version will appear in the Philosophical Transactions B Special Issue on Attention and Consciousness, edited by Peter Fazekas (in press, to be published 2018). The shorter version will have links/supplementary materials so that it covers all contents presented in this preprint publication. 


\begin{abstract}
:
Whether conscious perception requires attention remains a topic of intense debate. While certain complex stimuli such as faces and animals can be discriminated outside the focus of spatial attention, many simpler stimuli cannot. Because such evidence was obtained in dual-task paradigms involving no measure of subjective insight, it remains unclear whether accurate discrimination of unattended complex stimuli is the product of automatic, unconscious processing, as in blindsight, or is accessible to consciousness. Furthermore, these paradigms typically require extensive training over many hours, bringing into question whether this phenomenon can be achieved in naive subjects. We developed a novel dual-task paradigm incorporating confidence ratings to calculate metacognition and adaptive staircase procedures to reduce training. With minimal training, subjects were able to discriminate face-gender despite little or no top-down attentional amplification, while also displaying above-chance metacognitive accuracy. In contrast, the discrimination of simple coloured disks was significantly impaired and metacognitive accuracy collapsed, even in a partial-report condition. In a final experiment we used blended face/disk stimuli and confirmed that face-gender but not colour orientation can be discriminated in the dual-task. Our results show direct evidence for metacognitive conscious access in the near absence of attention for complex, but not simple, stimuli.
\end{abstract}




\section{Introduction}

The perplexing co-dependency between attention and consciousness has been

the subject of philosophical and scientific debate for well over a century (Wundt, 1874). One feature of this debate that has risen to prominence in recent years concerns the necessity of top-down attentional amplification for conscious perception (the necessity claim) (Cohen, Dennett, \& Kanwisher, 2016; Dehaene, 2014; Jennings, 2015). While several noteworthy theories of consciousness remain divided on this claim, scientific enquiry has made progress in its attempts to independently manipulate top-down attention and visual consciousness using a variety of tasks and visual illusions (Tsuchiya \& Koch, 2016; van Boxtel, 2017; van Boxtel, Tsuchiya, \& Koch, 2010).

The relationship between visual consciousness and top-down attentional amplification has been primarily investigated with the dual-task paradigm (Braun \& Julesz, 1998; Braun \& Sagi, 1990; Sperling \& Dosher, 1986). In this paradigm, a subject's attention is spatially drawn to a very demanding central task at the same time as a secondary stimulus is briefly presented in the periphery. If performance on the peripheral task in this diverted attention condition is identical to that without the central task, top-down attention is claimed unnecessary for the peripheral task. This paradigm has been employed to examine the requirement of top-down attention for discriminating many categories of stimuli (see Sup Fig 1). For example, a simple, low-level visual distinction such as discriminating the orientation of a coloured shape can be $80 \%$ correct under full-attention and yet fall to chance (50\% correct) when attention is diverted, demonstrating a necessity of spatial attention (Lee, Koch, \& Braun, 1999; Reddy, Wilken, \& Koch, 2004).

Remarkably, however, performance for certain visual discriminations that seem intuitively more complex such as categorising face-gender or identifying animals and vehicles in natural scenes does not differ between the full and diverted attention conditions (F. F. Li, VanRullen, Koch, \& Perona, 2002; Reddy, Reddy, \& Koch, 2006; Reddy et al., 2004). These results suggest that for certain complex stimuli, attention may not be necessary for conscious perception, a result taken as empirical evidence for 
a dissociation between top-down attention and consciousness (Koch \& Tsuchiya, 2007; van Boxtel et al., 2010).

However, dual-task studies investigating such a dissociation are confronted with various criticisms that question this conclusion. Among these, we address the most critical four in our experiments. Firstly, dual-task experiments typically employ extensive training of thousands of trials. In the case of Reddy et al. (2004), training took between 6 to 12 hours before subjects achieved proficiency. This involved as many as 5760 training trials, more than the experiment itself. Training is known to influence the attentional requirements of the dual-task (Bherer et al., 2005; Braun, 1998; Joseph, Chun, \& Nakayama, 1997; Sigman \& Gilbert, 2000) and other paradigms including the attentional blink (Garner, Tombu, \& Dux, 2014; Verghese, Mattingley, Garner, \& Dux, 2017). Specifically, the extent to which tasks are influenced by inattention differs between highly trained and naive subjects, which poses critical limitations on the conclusions that can be drawn when such tasks are employed to examine the relationship between attention and consciousness (Braun, 1998; Joseph et al., 1997; Sigman \& Gilbert, 2000).

The second challenge concerns the nature of the control experiments employed in the studies listed above (F. F. Li et al., 2002; Reddy et al., 2006, 2004). In these experiments and under the dual-task condition, certain categories of stimuli such as rotated letters or bisected disks are shown to be impossible to discriminate at above chance levels (Tsuchiya \& Koch, 2016) (see Sup Fig 1). A typical account of this scenario, inattentional blindness (Mack \& Rock, 1998), suggests that without top-down attentional amplification these stimuli fail to reach consciousness. An alternative account is that the inability to discriminate such items is not perceptual but results from response interference. That is, without top-down attention, while subjects are responding to the central task, they forget the stimulus because peripheral representations decay very quickly. In other words, the standard dual-task paradigm cannot exclude a possibility of conscious visibility with rapid forgetting of those stimuli 
that result in chance-level performance under the diverted attention condition (Block, 2011; Lamme, 2010; Sperling, 1960; Wolfe, 1999).

The third criticism concerns the difference in attentional draw between different categories of stimuli. Of particular concern for the studies by Reddy and colleagues (2004; 2006), faces are known to strongly attract (bottom-up) attention, possibly due to the ecological importance and the presence of dedicated neural resources (Cerf, Frady, \& Koch, 2009; Fletcher-Watson, Findlay, Leekam, \& Benson, 2008; Johnson, Dziurawiec, Ellis, \& Morton, 1991). They capture attention in individuals as young as 6 weeks old (Cashon \& Cohen, 2003), and they impair processing of visual objects that are presented elsewhere at the same time (Bindemann, Burton, Hooge, Jenkins, \& de Haan, 2005; Theeuwes \& Van der Stigchel, 2006). Perhaps this attraction to faces accounts for why this category of stimulus can be discriminated in the dual-task while less salient items, such as bisected disks, are missed?

Finally, it remains unclear whether successful dual-task performance for categories such as face-gender is in fact conducted using information accessible to consciousness. Recent literature has accumulated considerable evidence of above-chance behavioural performance that is not accompanied with consciousness, such as blindsight (Persaud, Navindra, Peter, \& Alan, 2007; Stoerig \& Cowey, 2007; Weiskrantz, Warrington, Sanders, \& Marshall, 1974). Non-conscious stimulus processing has been observed not only for simple discriminations, but also for complex, high-level stimuli such as upright faces (Debner \& Jacoby, 1994; Y. Jiang, Costello, \& He, 2007; Yi Jiang, Yi, \& Sheng, 2006; Merikle \& Joordens, 1997) for reviews, see (Dehaene \& Changeux, 2011; Yang, Brascamp, Kang, \& Blake, 2014). Thus, achieving highly accurate dual-task performance in tasks with complex stimuli such as faces does not guarantee that these discriminations are performed consciously (Jennings, 2015). In fact, in combination with excessive training, there is reason to suspect that at least some aspects of successful discrimination under dual-task conditions may be a product of unconscious processing (Merikle \& Joordens, 1997). 
In this paper, we addressed these four criticisms by substantially improving the dual-task paradigm in four ways. First, we employed an adaptive staircase procedure (Watson \& Pelli, 1983) to reduce the amount of training typical of dual-task studies (Li et al., 2002; Reddy et al., 2004). Second, our partial report condition made both the central and peripheral targets task-relevant but required subjects make only one response per trial, removing the concern that peripheral target representations decay beyond reportability while subjects respond to the central task. Third, we accounted for the salience of faces by blending face and disk stimuli through transparency (alpha ( $\boldsymbol{\alpha})$ blending) and examining whether the colour orientation of the disk is reportable when the stimuli are co-located. Fourth, we directly assessed metacognitive insight for unattended peripheral stimuli, a signifier of conscious access, by quantifying trial-by-trial confidence ratings and perceptual awareness judgements as a function of task accuracy (Fleming \& Lau, 2014; Rosenthal, 2000; Song et al., 2011; Stein \& Peelen, 2017). With these improvements over previous approaches, we critically assessed the necessity of top-down attention for conscious access of stimuli with high and low complexity. 


\section{General Methods}

Subjects

Twenty-four subjects participated in our study, eight for each of our three experiments. Participant numbers were determined from those studies that employed the dual-task paradigm: typically between four to eight (F. F. Li et al., 2002; Reddy et al., 2006, 2004; VanRullen, Reddy, \& Koch, 2004). A power analysis (power of 0.8, assumed correlation of 0.5 , one-tailed t-test) based on observations from the disk task in Reddy et al. (2004) revealed that a sample size of 3 would be sufficient to find a difference comparable to or larger than their study. Subjects were recruited from the student and staff bodies of Monash University and were paid for their involvement in the study. All had normal or corrected-to-normal vision and provided informed written consent in accordance with the guidelines of the Monash University Human Research Ethics Committee and the recommendations of the Declaration of Helsinki.

\section{Apparatus}

All experiments were performed on a MacBook Pro laptop connected to a 22 inch SMI monitor approximately 60 centimeters from the subject. Refresh rate of the monitor was fixed at $60 \mathrm{~Hz}$ with $1680 \times 1050$ pixels screen resolution. The experiments were programmed and conducted using the Psychophysics toolbox extension for Matlab (Psychtoolbox-3; (Brainard, 1997)). 
a.

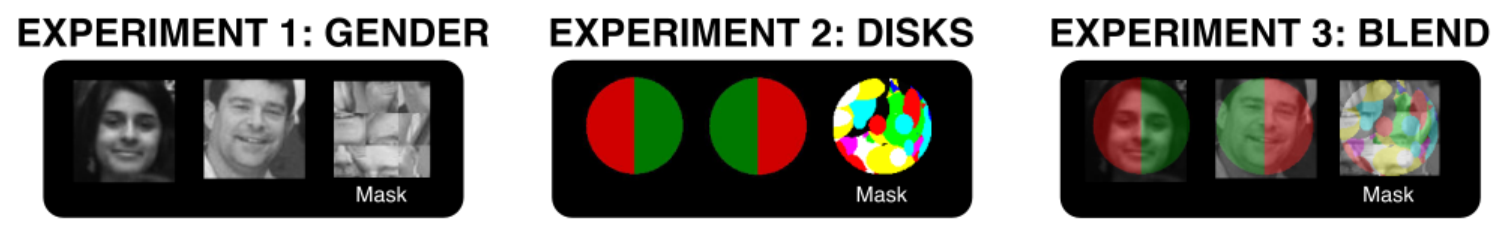

b.
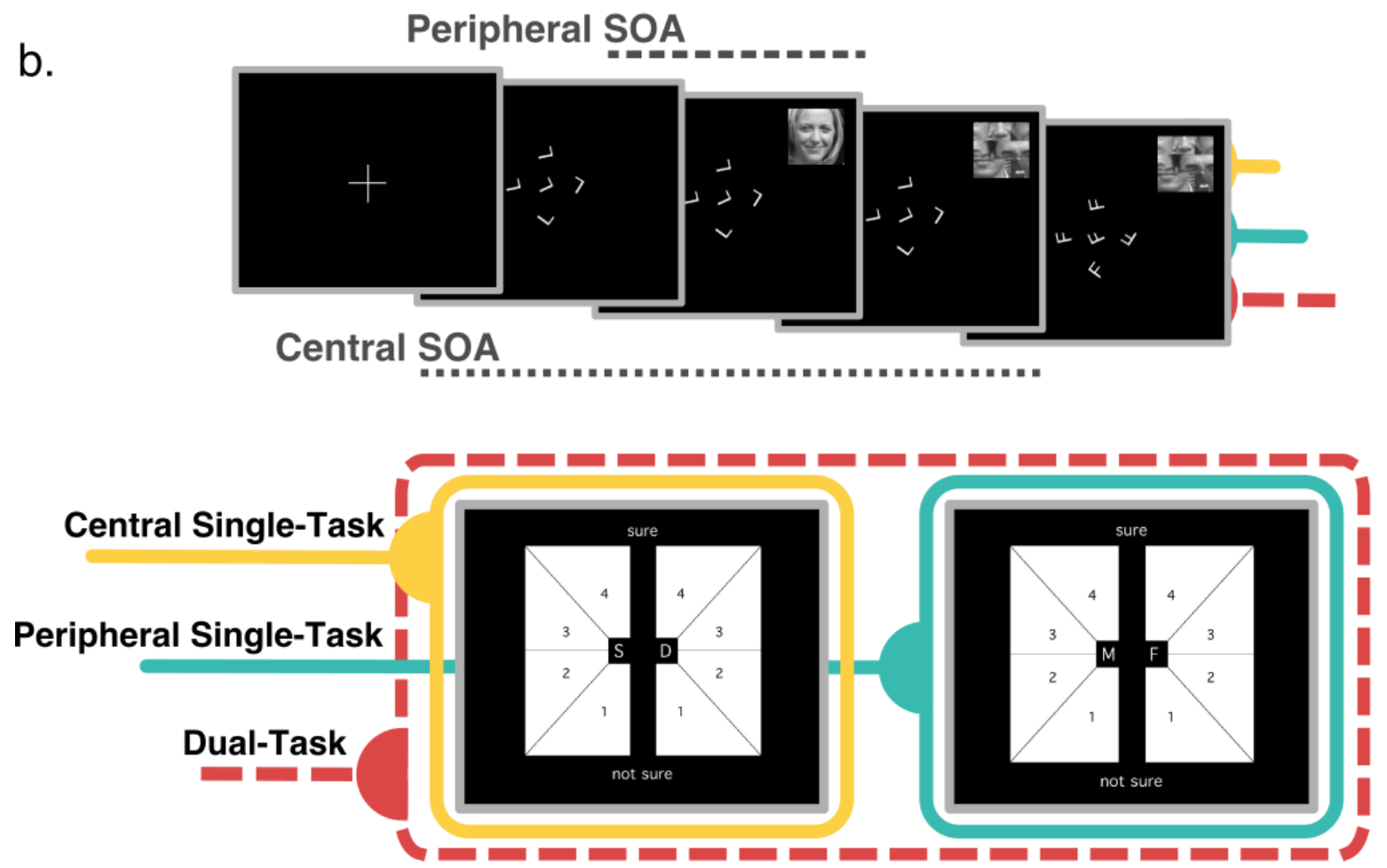

c.
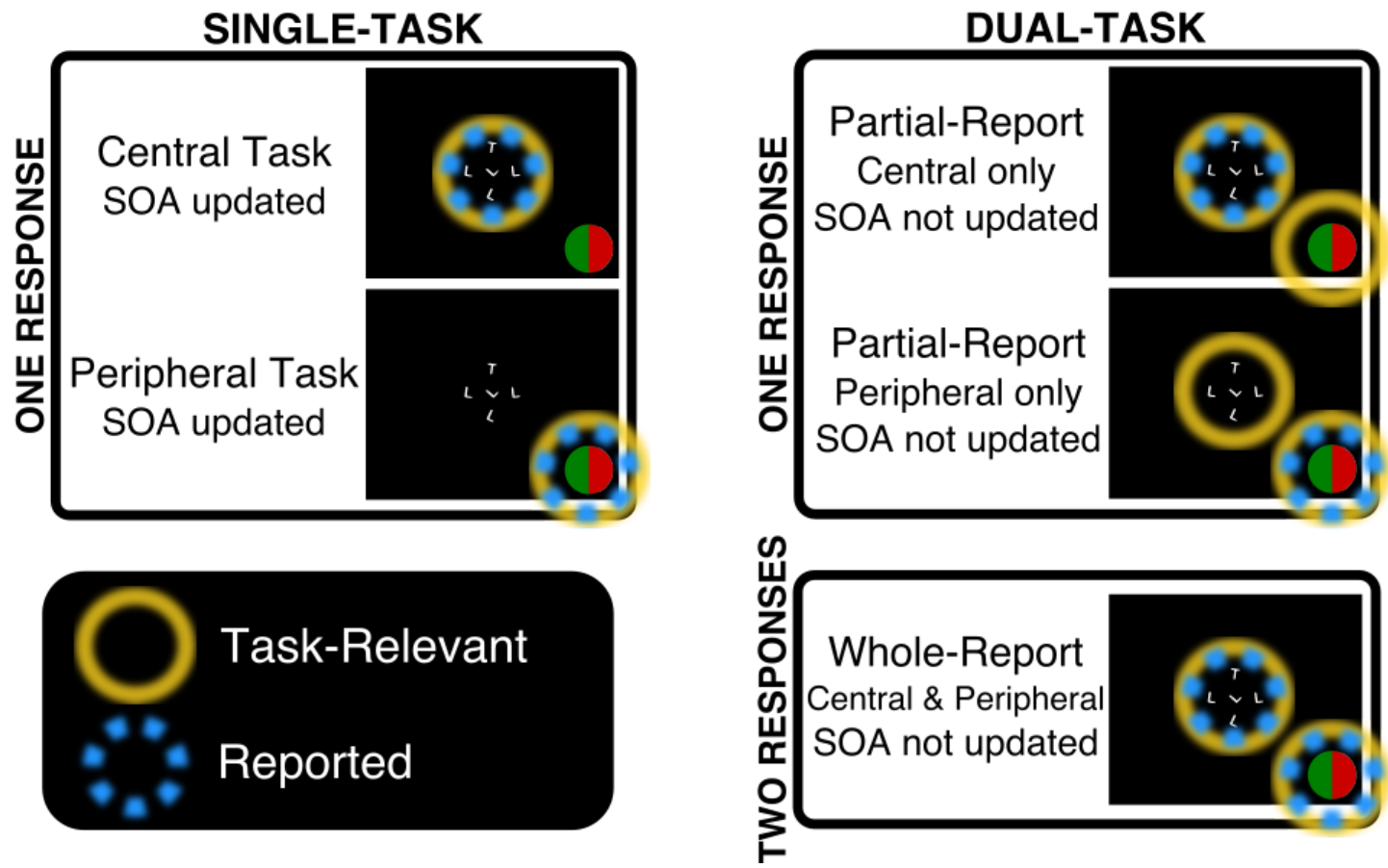
Figure 1. Stimuli and task structure. a) Stimuli used for Exp 1: gender discrimination, Exp 2: bisected disk discrimination, and Exp 3: blended face/disk discrimination. b) Trial sequence. After a variable period of fixation (200-400ms), five randomly rotated letters (Ls and/or Ts) are presented in the centre. After one frame $(16.7 \mathrm{~ms})$, a peripheral target (here, a female face) appeared in the periphery. Following a short delay, or Stimulus Onset Asynchrony (SOA), both central and peripheral targets were masked. In blocks involving the central task (the central single-task and dual-task), subjects reported on the letter stimuli at the centre of the display. Subjects made an $8 \mathrm{AFC}$ response with a single mouse-click, signalling their discrimination (' $S$ ' for same or ' $D$ ' for different) and 4-level subjective rating (confidence in Exp 1 and 2, perceptual awareness in Exp 3). In blocks involving the peripheral task (the peripheral single- and dual-task), subjects reported on the peripheral stimulus. Again, a single mouse-click was used for an 8AFC decision on face gender (Exp 1 and 3) or disk colour-orientation (Exp 2 and 3). c) Summary of partial- and whole- report conditions in Exp 2. During dual-task blocks, task relevance remained consistent but depending on the report condition either one or two responses were required.

\section{Procedure}

Dual-task experiments contrast performance in the single-task condition, where a central or a peripheral task is conducted in isolation, against that in the dual-task condition, where both the central and peripheral stimuli are task-relevant. The physical appearance of the experiment should be identical across these conditions, with the only difference being the task relevance of the stimuli (Figure 1c). Written instructions at the beginning of each block informed subjects which task was required.

\section{Single-task conditions}

In the central and peripheral single-task conditions, subjects were presented with one response screen and made a single eight-alternative-forced-choice (8AFC) report per trial (Figure 1b in green and yellow). Once subjects had signalled their readiness 
using a mouse-click, each trial began with the presentation of a fixation cross for 200 , 300 , or $400 \mathrm{~ms}$ with an equal probability for each. This was followed by the central stimulus and, on the next frame, the peripheral stimulus. Following a short temporal delay, central (or peripheral) SOA, the central (or peripheral) stimulus was masked (Figure 1b). SOAs were controlled such that discrimination accuracy for each stimulus in the single-task condition was held at 70\% (see QUEST description below).

\section{Dual-task condition with whole-report}

In the dual-task condition, both central and peripheral stimuli were task relevant. The presentation of visual items proceeded as above however subjects were required to make two responses per trial, first on the central then on the peripheral stimulus (Figure $1 \mathrm{~b}$ in red). We termed this dual-task condition 'whole-report' to contrast it with our 'partial-report' procedure in Experiment 2.

In order to contrast performance in the dual-task against the single-task conditions, SOAs were not updated during the dual-task. Instead, SOAs were fixed at the threshold duration defined by the preceding single-task block for each task type (see QUEST description). As is typical for this paradigm, we instructed subjects to prioritise performance for the central stimulus in the dual-task condition. We did not give subjects any feedback regarding their performance and did not inform them of the staircase procedure.

\section{Stimuli}

\section{Central letter discrimination}

The central stimulus for all experiments was a cluster of five uppercase characters presented in white, Helvetica script at 35 pixels text height (approximately $1^{\circ}$ visual angle on our setup) each rotated at a randomly selected angle. The coordinates of these five letters were fixed, one presented centrally at fixation and the remaining four located directly above, below and both sides of this point, approximately $3^{\circ}$ from fixation. These five letters were either all the same (all ' $T$ ' or all ' $L$ '), or contained a single differing character (i.e., one ' $T$ ' among four 'L's and vice versa). An uppercase 
letter ' $F$ ' individually masked each character for the remainder of the trial following a short, temporal delay that was adjusted to achieve $70 \%$ discrimination accuracy across central, single-task blocks (see QUEST staircase procedure). This letter discrimination task has proven effective in maintaining the focus of attention at the fixational point, leaving little or no spatial attention available at the periphery (Lee et al., 1999; F. F. Li et al., 2002; Reddy et al., 2006, 2004).

\section{Peripheral discrimination}

The peripheral stimulus categories consisted of faces (Exp 1), disks (Exp 2), or blended face/disks (Exp 3) (see Figure 1a). On each trial, one such stimulus subtending $2.5^{\circ}$ of visual angle was displayed at the periphery. This peripheral stimulus was randomly positioned at one of four locations centred on the corners of an imaginary rectangle $8^{\circ} \times 10^{\circ}$ of visual angle in dimensions. After a short temporal delay, a mask replaced the peripheral stimulus (see Figure 1a). This delay was adjusted such that single-task discrimination was held at $70 \%$ accuracy (see QUEST staircase procedure and Figure 1b).

\section{AFC response screen}

Mask presentation was followed with the display of a response screen comprised of eight evenly split segments (see Figure $1 \mathrm{~b}$ ). With a single mouse click, this screen allowed subjects to register their 2AFC discrimination response as well as a 4-level subjective rating. Prior to the experiment, and during practice, subjects were verbally instructed to express their confidence from a complete guess (rating 1) to certainty (rating 4) in Experiment 1 and 2 or perceptual awareness from complete invisibility

(rating 1 ) to complete visibility (rating 4 ) in Experiment 3 . Verbal descriptors for ratings 2 or 3 were not made explicit, however, the experimenter encouraged subjects to fix these criterions across the sessions as best as they could, and use all four levels. The labels "sure" and "not sure" were displayed at the top and bottom of the screen to remind subjects of the scale of subjective rating.

At the centre of the display we presented the discrimination options. For the central stimuli, subjects indicated whether the target letters they had seen were all the 
same ('S') or one was different ('D') by clicking on one of the confidence segments on the side with the labels either 'S' or ' $D$ ' (Figure 1b). The 'S' option was always on the left. For the peripheral face-gender discrimination (Experiment 1 and 3), subjects indicated whether the target was either male ('M') or female ('F'). The ' $M$ ' option was always on the left. For the peripheral coloured disk stimuli (Experiment 2 and 3), the discrimination options were substituted with images of red-green and green-red disks (the red-green option on the left) and subjects selected a segment to indicate their percept and confidence.

\section{QUEST staircase procedure and reduced training}

Previous dual-task studies employed large amounts of training to stabilise subjects' performance at the threshold SOAs (Braun \& Julesz, 1998; F. F. Li et al., 2002; Reddy et al., 2006, 2004). For example, in Li et al (2002) training is described as usually taking "more than 10 hours (12,000 trials of all tasks combined)". Reddy and colleagues (Braun \& Julesz, 1998; F. F. Li et al., 2002; Reddy et al., 2006, 2004) trained subjects until they achieved $80 \%$ accuracy for the central letter discrimination task with an SOA below $250 \mathrm{~ms}$ for an entire one hour session. This procedure took "between 6 and 12 hours per subject". A separate experiment by Reddy and colleagues (2006) trained subjects for only 2 hours but all had participated in Li et al. (2002) so had approximately 10 hours of prior exposure to the central letter discrimination task (Braun \& Julesz, 1998; F. F. Li et al., 2002; Reddy et al., 2006, 2004).

To generalise conclusions of the dual-task paradigm into an untrained population, we reduced training to a minimal level by rapidly and robustly setting SOAs that yielded threshold performance levels equated between subjects. SOAs were adjusted on a trial-by-trial basis during single-task blocks using the Quick Estimate of Threshold (QUEST) adaptive staircase procedure (Watson \& Pelli, 1983). The initial SOA for central targets was $500 \mathrm{~ms}$ and $250 \mathrm{~ms}$ for peripheral stimuli. We set the $\beta$ parameter for QUEST to be 2 and the standard deviations to be $70 \%$ of the respective initial SOA during training. Once training was complete, the standard deviation 
parameter was reduced to $50 \mathrm{~ms}$ for both central and peripheral stimuli. On each trial of the respective single-task block we updated either the central or peripheral SOA such that discrimination performance was fixed at $70 \%$ correct for that condition. To contrast performance in the single-task conditions against the dual-task we did not update SOAs in the dual-task (Figure 1c). Central SOA in the dual-task was drawn from the preceding single-task central block and vice versa for the peripheral SOA.

Training in our experiments only took approximately 20 minutes, with subjects completing two single-task central blocks followed by two single-task peripheral blocks. This procedure reduced 12 hours of training by more than $97 \%$.

\section{Data analysis}

\section{Objective Performance: Type 1 AUC}

To estimate subjects' objective discrimination accuracy regardless of response criterion, we adopted signal detection theory (Macmillan \& Creelman, 2005) to calculate Type 1 performance. To construct a Type 1 receiver operating characteristics (ROC) curve, we regarded a trial in which the correct response was either 'same', 'male', or 'red-green' as a signal-present trial. Conversely, trials in which the correct response was either 'different', 'female', or 'green-red' were regarded as signal-absent. Starting from the most stringent criterion, a signal-present response with confidence 4 was defined as a 'hit' in a signal-present trial and 'false alarm' in a signal-absent trial. Then, in the second most stringent criterion, we regarded a signal-present response with confidence 4 and 3 in a signal-present trial as a hit and in a signal-absent trial as a false alarm, and so on. With the most lenient criterion, we considered any signal-present response and signal-absent response with confidence 1, 2 and 3 in a signal-present trial as hit and in a signal-absent trial as false alarm. Thus, we shifted the criterion in 7 steps to obtain a 7-inflection ROC curve. The area under this ROC curve (Type 1 AUC) was then computed to provide a non-parametric estimate of objective accuracy for each attention condition (Chen, Mundy, \& Tsuchiya, 2016; Kaunitz, Rowe, \& Tsuchiya, 2016; Wilimzig, Tsuchiya, Fahle, Einhäuser, \& Koch, 2008). 


\section{Metacognitive accuracy: Type 2 AUC}

To examine whether subjects categorise stimuli using information accessible to consciousness, we quantified metacognitive accuracy as Type 2 discrimination performance using signal detection theory (Fleming \& Lau, 2014; Kunimoto, Miller, \& Pashler, 2001; Song et al., 2011; Stein \& Peelen, 2017). Using the signal conventions described above, we first categorised trials as correct or incorrect. Correct responses were those where subjects made a signal-present side response in a signal-present trial or signal-absent side response in a signal-absent trial, regardless of the level of confidence. Otherwise, we regarded that trial as incorrect.

Trials were then classified according to confidence. First, we regarded a correct trial (regardless of whether the signal was present or not) with confidence rating of 4 as 'hit' and an incorrect trial with confidence 4 as 'false alarm', and so on. We shifted the criterion in 3 steps to obtain a 3-inflection ROC curve. The area under the resulting ROC curve (Type 2 AUC) was then adopted as a non-parametric estimate of metacognitive accuracy for each attention condition (Fleming \& Lau, 2014; Kaunitz et al., 2016; Macmillan \& Creelman, 2004). A similar process was used for the perceptual awareness judgements in Experiment 3 with the confidence ratings substituted for a perceptual awareness scale (PAS).

\section{Linear mixed effects (LME) metacognitive analysis}

We used linear mixed-effect (LME) analysis to examine if trial-by-trial subjective ratings (i.e., confidence ratings or PAS) are affected by spatial attention and task accuracy. We used the Ime4 package (Bates, Mächler, Bolker, \& Walker, 2015) within R (R Development Core Team, 2014) to model subjective ratings as our dependent variable (DV) with the fixed effects of attention, correctness, and their interaction. As a random effect we included intercepts for subjects. As a significance test, we performed likelihood ratio tests between the full model and a reduced model that excluded the factor of interest. In the case of a significant interaction effect, we subset data into each level of the attention or correctness factors and performed a likelihood ratio test against a null model with the random intercept for subjects only. 


\section{Tradeoff analysis}

When performance measures for both central and peripheral tasks under the dual-task condition are the same as those under their respective single-task conditions, discrimination for the peripheral task is considered not to rely on top-down attentional amplification (Koch \& Tsuchiya, 2007; Reddy et al., 2004; van Boxtel et al., 2010). When performance is not identical between the conditions, we need to consider tradeoff between the tasks.

In Figure 2, we plot hypothetical single-task performance for the central (dark blue circle) and peripheral (light blue circle) task on the $x$ - and $y$ - axes, with the origin corresponding to chance-level performance for each task. We plot a hypothetical dual-task performance with its $\mathrm{x}$ - and $\mathrm{y}$-coordinate corresponding to the central and peripheral performance in the dual-task condition (black circles within axes). If there is no tradeoff, the dual-task performance should locate at the corner (black circle highlighted in red). The line connecting the two single-task points (black diagonal) represents complete tradeoff between the tasks. To quantify the level of tradeoff we calculate the trigonometric altitude, or the shortest distance between the observed dual-task performance (black circle highlighted in salmon) and complete tradeoff line. We define the actual trigonometric altitude (salmon line, TA $^{\text {actual) }}$ ) as the observed altitude as a proportion of the altitude for no-tradeoff dual-task performance (red line, $\left.T A^{\text {theory }}\right)$. 


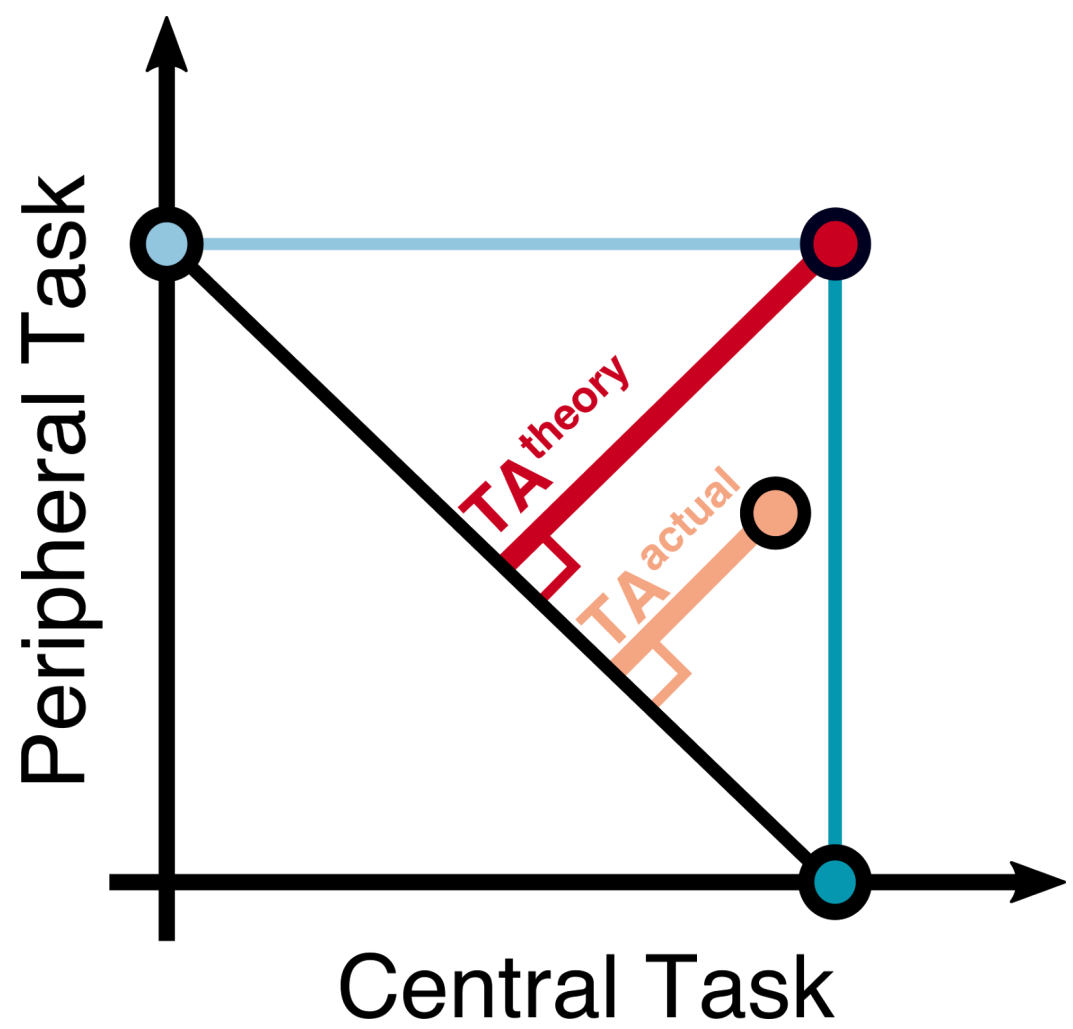

Figure 2. Schematic of tradeoff analysis. $X$ - and $y$ - axes are for central and peripheral performance. Single-task performance is plotted on the $x$ - or $y$-axis. When there is no tradeoff, the performance of cental and peripheral tasks in the dual-task condition locates in the top right (TA ${ }^{\text {theory }}$ of 1.00, the black circle with red highlight). When there is tradeoff, we quantify it as the trigonometric altitude for the observed dual-task as a proportion of TA ${ }^{\text {theory }}\left(\right.$ e.g., TA $^{\text {actual }}$ of 0.47 indicated by salmon). 


\section{Experiment 1: gender discrimination}

Our first experiment examined whether gender discrimination in the near absence of attention was associated with conscious access. In addition, we employed a staircase procedure to greatly reduce the amount of training.

Methods

Eight subjects (3M, 5F, ages 18-34) took part in Experiment 1. The procedure for the experiment was identical to our general method but employed grayscale photographs of human faces as the peripheral stimulus.

A set of 65 male and 65 female faces were selected from natural crowd scenes, details of which are described elsewhere (Kaunitz et al., 2016). All were facing forward with major features (i.e. eyes, mouth, nose) clearly visible. To generate masking textures, we used 20 male and 20 female faces (out of 130 used for the experiment), each cut into $3 \times 3$ squares of equal size and rearranged randomly without rotation or flipping (Figure 1a). Faces and masks were randomly selected on each trial.

\section{Data collection}

Data collection took place over 3 sessions on 3 consecutive days. The first session consisted of training (i.e., 2 blocks $x 48$ trials of the single-central-letter task, 2 blocks $\times 48$ trials of the single-peripheral-gender task and 20 trials of the dual-task), followed by two runs of the experiment. Each run comprised one block each of the single-central, single-peripheral, and the dual-task whole-report condition. The order of these blocks in each run was randomised. SOAs were updated during each single-task block and these updated SOAs were used for the dual-task block that immediately followed. Each block consisted of 48 trials. The second and third sessions skipped training and consisted of 3 experimental runs. This resulted in 8 experimental runs overall, and thus 8 blocks for each condition (single-central, single-peripheral, dual-task) per subject. 
$\underline{\text { Results }}$

\section{Face-gender discrimination in near absence of attention}

Even with our minimal training procedure, we largely replicated previous findings (Reddy et al., 2006, 2004) (Figure 3A, Table 1). Objective performance (Type 1 AUC) for the peripheral face-gender and central letter tasks were much higher than chance ( 0.75 and 0.76 , respectively) when they were performed simultaneously in the dual-task condition. Dual-task performance was slightly worse than each respective single-task condition ( 0.77 and 0.80$)$. These differences were statistically significant $\left(p=.039, \eta_{p}^{2}\right.$ $=.037$ for peripheral and $\mathrm{p}=.025, \eta_{p}^{2}=.044$ for central) according to a two-way within-subject ANOVA (attention condition [single- vs dual-task] and block as factors). Neither the interaction or main effect of block was significant ( $p>.25$, see Table 1). The $\mathrm{TA}^{\text {actual }}$ for objective performance was 0.77 , indicating almost no tradeoff.

Table 1. Objective Performance in Experiment 1

\begin{tabular}{|c|c|c|c|c|c|}
\hline \multirow[b]{2}{*}{ EXP 1} & \multirow[b]{2}{*}{ ST } & \multirow[b]{2}{*}{ DT } & \multicolumn{3}{|c|}{ Two-way ANOVA p-values } \\
\hline & & & attention [ST vs DT] & block & attention $\mathrm{x}$ block \\
\hline Faces & $.78 \pm .013$ & $.75 \pm .012$ & 0.039 & $>.25$ & $>.25$ \\
\hline \multirow[t]{3}{*}{ Letter } & $.80 \pm .014$ & $.76 \pm .027$ & 0.025 & $>.25$ & $>.25$ \\
\hline & \multicolumn{3}{|c|}{$\mathrm{TA}^{\text {actual }}$} & & \\
\hline & mean \pm sem & $p$ against 0 & p against 1 & & \\
\hline Letter + Faces & $.77 \pm .063$ & $<.001$ & 0.007 & & \\
\hline
\end{tabular}

\section{Confidence remains stable in the near absence of attention}

As a subjective measure, we asked subjects to rate trial-by-trial confidence on their discrimination in Experiment 1 (Figure 3B, Figure 4A and Table 2). We conducted LME analysis to examine the relationship between attention and correctness on trial-by-trial confidence ratings (see LME analysis in Methods) (We also performed the analyses including block as a factor, but block was never significant in any analysis 
across all experiments and measures (all $p>.25$ ), thus we will not report it further). For the peripheral face-gender task, the main effect of attention did not reach significance ( $p>.05$ for either correct or incorrect trials), but that for correctness was highly significant $\left(x^{2}(1)>110\right.$ and $p<.001$ for both the single- and dual-task). The interaction between attention and correctness was also significant, though the effect size was small $\left(x^{2}(1)=4.26, p=.039\right)$.

Table 2. Mean confidence ratings for correct and incorrect trials in Experiment 1

\begin{tabular}{|c|c|c|c|}
\hline EXP 1 & ST & DT & \\
\hline Faces (correct) & $2.6 \pm .18$ & $2.5 \pm .19$ & $\begin{array}{c}\text { Attention: } \\
\mathrm{x}^{2}=3.8, \mathrm{p}=.053\end{array}$ \\
\hline \multirow[t]{3}{*}{ Faces (incorrect) } & $2.1 \pm .19$ & $2.2 \pm .18$ & $\begin{array}{c}\text { Attention: } \\
x^{2}=1.9, p=.171\end{array}$ \\
\hline & $\begin{array}{c}\text { Correctness: } \\
x^{2}=174.0, p<.001\end{array}$ & $\begin{array}{c}\text { Correctness: } \\
x^{2}=112.9, p<.001\end{array}$ & $\begin{array}{c}\text { Interaction: } \\
x^{2}=4.26, p=.039\end{array}$ \\
\hline & ST & DT & \\
\hline Letter (correct) & $2.9 \pm .16$ & $2.8 \pm .16$ & $\begin{array}{c}\text { Attention: } \\
x^{2}=13.9, p<.001\end{array}$ \\
\hline \multirow[t]{2}{*}{ Letter (incorrect) } & $2.5 \pm .19$ & $2.5 \pm .20$ & $\begin{array}{c}\text { Attention: } \\
x^{2}=.003, p>.25\end{array}$ \\
\hline & $\begin{array}{c}\text { Correctness: } \\
x^{2}=182.5, p<.001\end{array}$ & $\begin{array}{c}\text { Correctness: } \\
x^{2}=120.5, p<.001\end{array}$ & $\begin{array}{c}\text { Interaction: } \\
x^{2}=4.15, p=.042\end{array}$ \\
\hline
\end{tabular}

\section{Metacognitive accuracy in the near absence of attention}

That confidence ratings broadly correspond with subjects' accuracy implies that subjects had metacognitive insight into their decisions. We examined this relationship quantitatively by computing metacognitive accuracy (measured as Type 2 AUC, see General Methods) (Figure $3 \mathrm{C}$ and Table 3). While metacognitive accuracies for both peripheral faces and central letters were well above chance during the dual-task (0.59 and 0.60 ), they were significantly lower than those in the single task (0.62 and 0.64 , 
two-way within-subject ANOVA: main effect of attention $\left.\mathrm{p}<.05, \eta_{p}^{2}=.036\right)$. TA ${ }^{\text {actual }}$ was 0.50 , reflecting metacognitive accuracy roughly half that of single-task equivalence.

Table 3. Metacognitive accuracy in Experiment 1

\begin{tabular}{|c|c|c|c|c|c|}
\hline \multirow[b]{2}{*}{ EXP 1} & \multirow[b]{2}{*}{ ST } & \multirow[b]{2}{*}{ DT } & \multicolumn{3}{|c|}{ Two-way ANOVA p-values } \\
\hline & & & attention [ST vs DT] & block & attention $\mathrm{x}$ block \\
\hline Faces & $.62 \pm .017$ & $.59 \pm .014$ & 0.042 & 0.155 & $>.25$ \\
\hline \multirow[t]{3}{*}{ Letter } & $.64 \pm .017$ & $.60 \pm .022$ & 0.016 & $>.25$ & $>.25$ \\
\hline & \multicolumn{3}{|c|}{ TA $^{\text {actual }}$} & & \\
\hline & mean \pm sem & $p$ against 0 & p against 1 & & \\
\hline Letter + Faces & $.50 \pm .123$ & 0.005 & 0.005 & & \\
\hline
\end{tabular}

Taken together, these results confirm that despite their attention being consumed by the central letter task, subjects exhibited near intact objective accuracy and confidence ratings on their peripheral face discriminations in the dual task. While some performance decrement was seen, the magnitude was small by Cohen's conventions. Given that subjects were minimally trained in our protocol (20 minutes), many causes may explain this slight deficit (e.g., motor coordination errors, confusion due to task switching across blocks), and rather it is remarkable that we found highly similar results to the original findings, which required training subjects for 6-12 hours. Further, no statistical analyses on performance measures showed significant effects of training (i.e., the main effect of block or interaction between block and attention), implying that our block-by-block SOA adjustment was successful in achieving stable performance. Our QUEST protocol was robust and stable enough that we saw no effects of block across any measures in Experiment 2 and 3 as well (thus they are not reported further).

While subjects retain the capacity to discriminate face-gender in the near absence of attention, their metacognitive insight into these discriminations was affected in the dual-task condition. This might be due to the slight dual-task tradeoff in objective accuracy and confidence ratings. We will return to this issue in our General Discussion. 
A
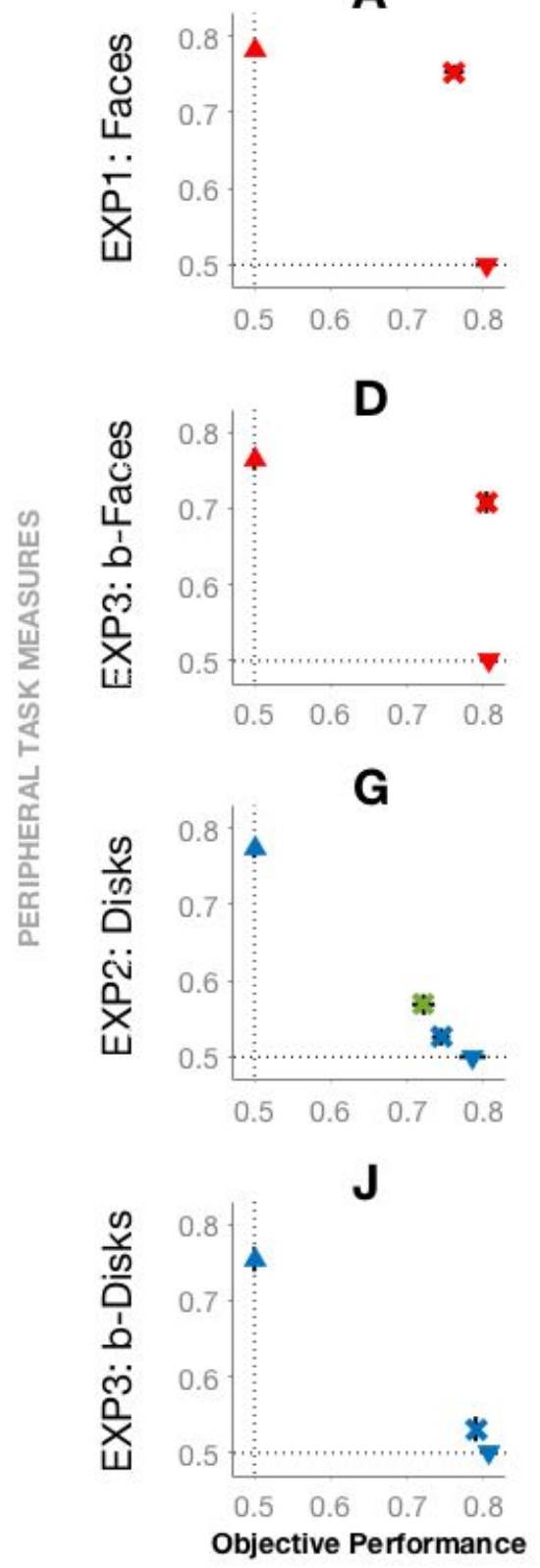

B
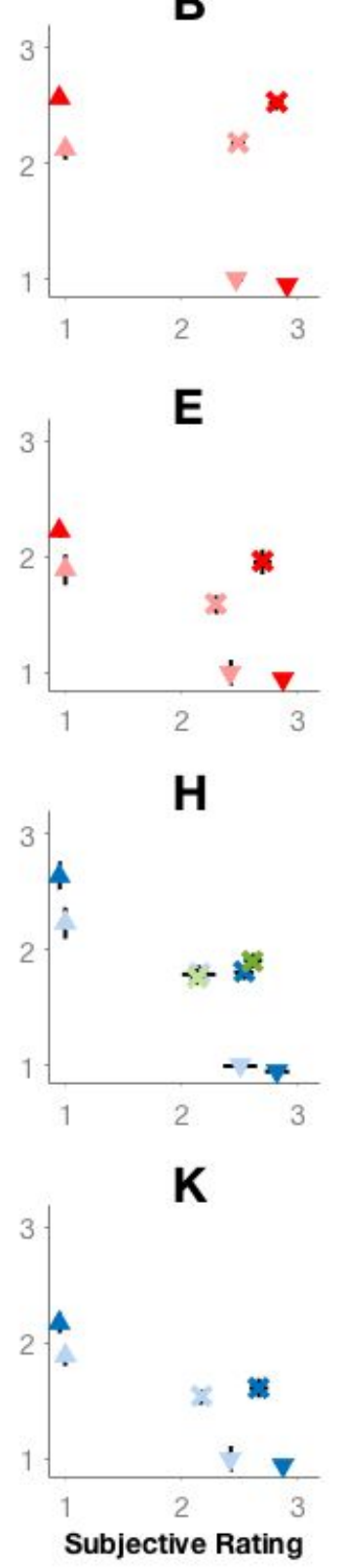
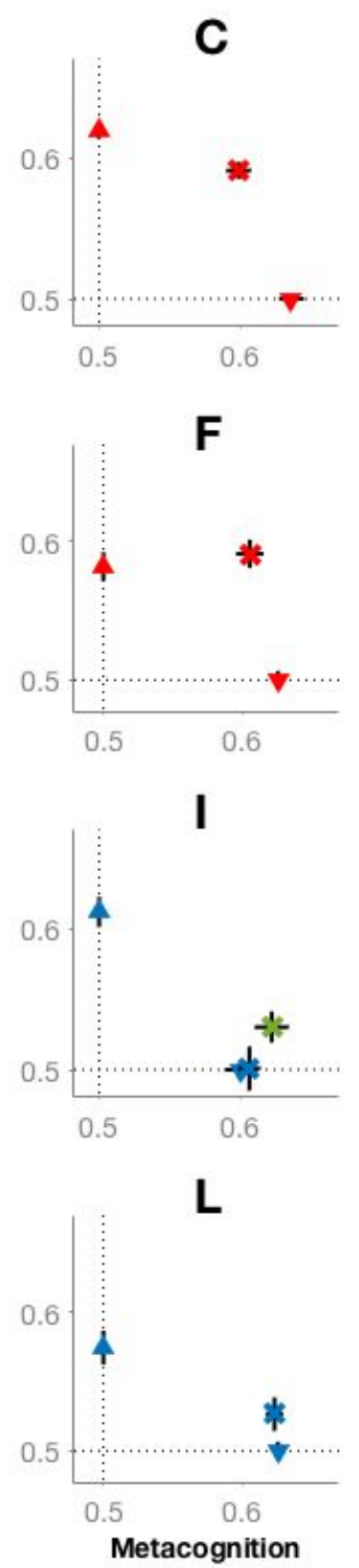

CENTRAL TASK MEASURES 
Figure 3. Conscious face-gender, but not disk-colour, discrimination is possible in the near absence of attention despite minimal training. Presented in red, the first (A-C) and second (D-F) row reflect discrimination of face-gender in Exp 1 and 3. Presented in blue, rows three (G-I) and four (J-L) reflect disk-colour discrimination in Exp 2 and 3. Exp 3 used a blended face and disk. Each column represents a performance measure; column 1 (A, D, G, J) is objective performance (Type $1 \mathrm{AUC}$ ) and column $3(\mathrm{C}, \mathrm{F}, \mathrm{I}, \mathrm{L})$ is metacognitive accuracy (Type 2 AUC). Column 2 reflects subjective ratings (confidence judgements in Exp $1(B)$ and $2(E)$, PAS in Exp 3 ( $H$ and $K)$ ). Subjective ratings are plotted separately for correct and incorrect trials (lighter shades reflect mean ratings for incorrect judgements). In all panels, measures along the $x$-axis refer to performance on the central letter task. Measures along the $y$-axis refer to performance on the peripheral task. Single-task performance are plotted as upright (for peripheral tasks) and inverted (for central task) triangles near their respective axes. Dual-task performance are plotted as ' $x$ '. Additional green markers are included for Exp 2 to reflect results from the partial-report procedure. Error bars signify within-subjects standard error of the mean (Cousineau, 2005; O'Brien \& Cousineau, 2014). 

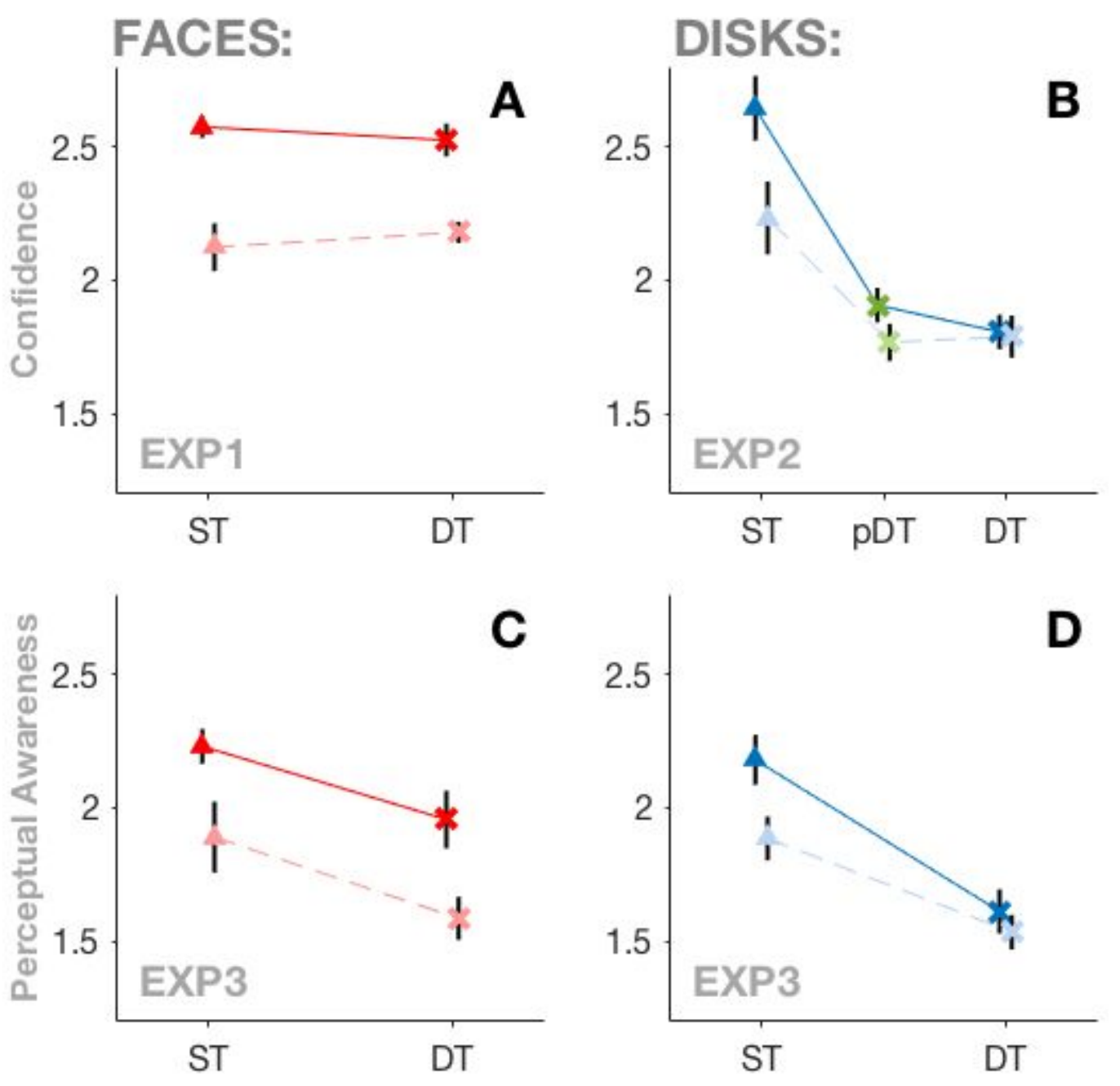

Figure 4. Subjective ratings for peripheral stimuli as a function of attention and correctness. Top row represents confidence ratings for Exp 1 (A) and 2 (B) respectively. Bottom row represent perceptual awareness scales (PAS) in Exp 3 (C for faces, $D$ for disks). Lighter shading indicates mean subjective rating for incorrect responses and additional green markers in (B) for partial-report. Error bars reflect within-subjects standard error of the mean (Cousineau, 2005; O'Brien \& Cousineau, 2014). 


\section{Experiment 2: disk discrimination}

In Experiment 2 we employed the dual-task design for coloured disks, a stimulus category regarded as indiscriminable when selective attention is diverted (Lee et al., 1999; F.-F. Li, van Rullen, Koch, \& Perona, 2005; Reddy et al., 2004). To test if response interference can explain this phenomenon, we used a partial report procedure (Figure 1c, see Methods for Exp 2).

\section{Methods}

Eight new subjects (4 females, ages 19-38) participated in Experiment 2. Methods for this experiment were identical to our general methods apart from the inclusion of the partial-report condition and the use of coloured disks as the peripheral stimulus (Figure 1a). Disks were masked by one of ten circular, multi-coloured Mondrian patches precomputed before the experiment (Figure 1a).

\section{Dual-task condition with partial report}

In dual-task partial-report blocks, central and peripheral stimuli presentation proceeded as usual but subjects were required to respond to just the central or the peripheral stimulus on a given trial. Participants did not know in advance which stimulus to respond, thus both tasks remained relevant while the report demands were equivalent to the single-task conditions (Figure 1c).

\section{Data collection}

As in Experiment 1, training and testing were conducted over the course of 3 sessions on 3 consecutive days. In session one, training involved 2 blocks of 30 trials for the single-central letter task and single-peripheral disk task (120 trials in total) and 20 trials of the dual-task under whole-report conditions. No separate training was given under partial-report conditions apart from verbal and written instructions at the beginning of each block. 
After training, two runs of the main experiment followed in the first session. Each run contained 4 blocks of 30 trials in length: one block of single-central-letter task (updated central SOA), one block of single-peripheral-disk task (updated peripheral SOA), one block of whole-report dual-task (fixed SOAs), and one block of partial-report dual-task (fixed SOAs) (Figure 1c). The order of these 4 experimental blocks was randomised within each run. Sessions two and three consisted of three runs resulting in a total of 8 blocks of each condition per subject.

\section{$\underline{\text { Results }}$}

\section{Disks cannot be discriminated in dual-task conditions even with partial report}

In Experiment 2, subjects discriminated the orientation of coloured disks in the single-task, as well as the dual-task under both whole- and partial-report conditions (Figure 3G, Table 4). Replicating previous studies (Lee et al., 1999; Reddy et al., 2004), objective accuracy (Type $1 \mathrm{AUC}$ ) for the peripheral disk task was near chance in the dual-task in both traditional whole- and our novel partial-report conditions ( 0.53 and 0.57 , respectively) when the central letter task was prioritised (0.74 and 0.72 ). In contrast, both disk and letter tasks could be performed well above chance in the single-task conditions ( 0.77 and 0.79 , respectively). Critically, we found complete tradeoff $\left(T^{\text {actual }}=-0.04\right.$ and 0.02$)$, for both the whole- and partial-report conditions (Table 4). A follow-up t-test confirmed no difference between these conditions (paired-sample t-test: $t(7)=-.608, p>.25,95 \% \mathrm{Cl}[-.290, .171])$. 
Table 4. Objective Performance in Experiment 2

\begin{tabular}{|c|c|c|c|}
\hline EXP 2 & ST & pDT & wDT \\
\hline Disks & $.77 \pm .012$ & $.57 \pm .020$ & $.53 \pm .011$ \\
\hline Letter & $.79 \pm .016$ & $.72 \pm .027$ & $.75 \pm .020$ \\
\hline & & \multicolumn{2}{|c|}{ TA $^{\text {actual }}$} \\
\hline & mean \pm sem & p against 0 & p against 1 \\
\hline Letter + Disks (whole-report) & $-.04 \pm .055$ & $>.25$ & $<.001$ \\
\hline Letter + Disks (partial-report) & $.02 \pm .100$ & $>.25$ & $<.001$ \\
\hline
\end{tabular}

Confidence in discriminations for both letter and disk tasks is reduced when attention is diverted

As in Experiment 1, we asked subjects to rate confidence in their discrimination judgements (Figure 3H, Figure 4B, and Table 5). To examine the relationship between attention and correctness on confidence ratings in Experiment 2 we conducted LME analysis (see LME analysis in Methods). For peripheral disk discriminations, the full model with interaction term differed substantially from the reduced model $\left(x^{2}(2)=68.9\right.$, $p<.001)$. Subsetting data into correct and incorrect judgements revealed significant main effects of attention $\left(x^{2}(2)=769.9, p<.001\right.$ and $x^{2}(2)=146.1, p<.001$ respectively). Subsetting by attention condition, the main effect of correctness reached significance for the single-task $\left(x^{2}(1)=111.4, p<.001\right)$ and dual-task partial-report $\left(x^{2}(1)=13.6, p<.001\right)$ conditions but not dual-task whole-report $\left(x^{2}(1)=1.3, p>.25\right)$. This confirmed that the relationship between correctness and confidence ratings was moderated by attention. Higher confidence corresponded with correct judgements when subjects fully attended to the disk task but this relationship was largely extinguished when attention was diverted (Figure 4B). 
Table 5. Mean confidence ratings for correct and incorrect trials in Experiment 2

\begin{tabular}{|c|c|c|c|c|}
\hline EXP 2 & ST & pDT & wDT & \\
\hline Disks (correct) & $2.6 \pm .24$ & $1.9 \pm .16$ & $1.8 \pm .17$ & $\begin{array}{c}\text { Attention: } \\
\mathrm{x}^{2}=796.9, \mathrm{p}<.001\end{array}$ \\
\hline \multirow[t]{2}{*}{ Disks (incorrect) } & $2.2 \pm .24$ & $1.8 \pm .14$ & $1.8 \pm .18$ & $\begin{array}{c}\text { Attention: } \\
x^{2}=146.1, p<.001\end{array}$ \\
\hline & $\begin{array}{c}\text { Correctness: } \\
x^{2}=111.4, p<.001\end{array}$ & $\begin{array}{l}\text { Correctness: } \\
x^{2}=13.6, p<.001\end{array}$ & $\begin{array}{l}\text { Correctness: } \\
x^{2}=1.3, p>.25\end{array}$ & $\begin{array}{c}\text { Interaction: } \\
\mathrm{x}^{2}=68.98, \mathrm{p}<.001\end{array}$ \\
\hline & ST & pDT & wDT & \\
\hline Letter (correct) & $2.8 \pm .22$ & $2.6 \pm .16$ & $2.5 \pm .17$ & $\begin{array}{c}\text { Attention: } \\
x^{2}=90.9, p<.001\end{array}$ \\
\hline \multirow[t]{2}{*}{ Letter (incorrect) } & $2.5 \pm .24$ & $2.1 \pm .16$ & $2.2 \pm .18$ & $\begin{array}{c}\text { Attention: } \\
x^{2}=78.0, p<.001\end{array}$ \\
\hline & $\begin{array}{l}\text { Correctness: } \\
x^{2}=83.0, p<.001\end{array}$ & $\begin{array}{c}\text { Correctness: } \\
x^{2}=147.8, p<.001\end{array}$ & $\begin{array}{c}\text { Correctness: } \\
x^{2}=122.2, p<.001\end{array}$ & $\begin{array}{c}\text { Interaction: } \\
x^{2}=6.42, p=.040\end{array}$ \\
\hline
\end{tabular}

Metacognitive accuracy for disk discriminations reduces to chance in the near absence of attention

Metacognitive accuracy for the peripheral disk task is summarised in Figure $3 \mathrm{l}$ and Table 6. It fell near chance level under the dual task, in both the partial (0.53) and whole-report (0.50) conditions, though it was much above chance in the single-task condition (0.61). As we expected from our instruction to prioritise the central task, we found metacognitive accuracy of the central task was similar across conditions $(\sim 0.61)$. In terms of tradeoff analysis, we observed complete tradeoff under the whole-report condition, but not in the partial-report condition (see Table 6). We will return to this and related problems regarding tradeoff analysis of metacognition in our General Discussion. 
Table 6. Metacognitive accuracy in Experiment 2

\begin{tabular}{|c|c|c|c|c|c|c|}
\hline \multirow[b]{2}{*}{ EXP 2} & \multirow[b]{2}{*}{ ST } & \multirow[b]{2}{*}{ pDT } & \multirow[b]{2}{*}{ wDT } & \multicolumn{3}{|c|}{ Two-way ANOVA p-values } \\
\hline & & & & $\begin{array}{c}\text { attention } \\
{[\mathrm{ST}, \mathrm{pDT}, \mathrm{wDT}]}\end{array}$ & block & $\begin{array}{l}\text { attention } \mathrm{x} \\
\text { block }\end{array}$ \\
\hline Disks & $.61 \pm .013$ & $.53 \pm .010$ & $.50 \pm .011$ & $<.001$ & $>.25$ & $>.25$ \\
\hline \multirow[t]{3}{*}{ Letters } & $.60 \pm .015$ & $.62 \pm .019$ & $.61 \pm .018$ & $>.25$ & 0.2 & $>.25$ \\
\hline & \multicolumn{3}{|c|}{$T^{\text {actual }}$} & & & \\
\hline & mean \pm sem & $p$ against 0 & $p$ against 1 & & & \\
\hline $\begin{array}{l}\text { Letters + Disks } \\
\text { (whole-report) }\end{array}$ & $.12 \pm .156$ & $>.25$ & $<.001$ & & & \\
\hline $\begin{array}{l}\text { Letters + Disks } \\
\text { (partial-report) }\end{array}$ & $.61 \pm .238$ & 0.036 & 0.155 & & & \\
\hline
\end{tabular}




\section{Experiment 3: blended face/disk discrimination}

In our final experiment we addressed the potential concern that gender discrimination of a face is made possible by its inherent salience (Cerf et al., 2009; Fletcher-Watson et al., 2008). To address this issue we developed a novel stimulus category by $\boldsymbol{\alpha}$-blending a face and a disk. If the saliency of faces explains our results, two aspects of the blended object, face-gender and colour orientation, should be equally discriminable under the dual task.

\section{Methods}

Eight subjects participated in Experiment 3 (3 females, ages 21-32). Methods were identical to our general methods except that an $\boldsymbol{\alpha}$-blended face/disk image was used as the peripheral stimulus (see $\alpha$ parameter staircasing). The disk aspect of this stimulus was identical (but lowered in contrast through transparency) to that described in Experiment 2. In order to create the face aspect, a novel set of 518 faces (half of them female) were selected from the natural crowd scenes cited above (Kaunitz et al., 2016). The addition of these extra faces ensured that subjects could not simply learn the face stimuli. In addition, we did not repeat a given face until every face had been presented (resulting in a maximum of 4 presentations of a given face across the entire experiment). As the masking stimuli, we generated approximately 3500 scrambled face textures and Mondrians, $\boldsymbol{\alpha}$-blended using the same technique as the blended face/disk stimulus.

\section{Adjustment of SOAs and $\alpha$ parameters during training}

To equate discrimination performance across subjects and between both face and disk aspects of the blended stimuli in the single-task condition, we employed QUEST to adjust SOA (with the same parameter settings as in Exp 1 and 2) or $\alpha$ parameters (QUEST parameter $\beta$ of 3 and standard deviation of 0.3 ).

As training, subjects completed 10 blocks of single-task (30 trials per block) and 2 blocks dual-task (20 trials per block). In blocks 1 and 2 we adjusted $\alpha$ values for the single-peripheral-disk and single-peripheral-face conditions. We adjusted SOAs in block 
3,4 , and 5 (single-central-letter, single-peripheral-disk, then single-peripheral-face conditions). This procedure for $\alpha$ and SOA staircasing was repeated in blocks 6 through 10. During single-task blocks with $\alpha$ adjustment, SOAs were fixed, and vice versa.

The $\alpha$ parameter of both the face and disk aspect of the blended stimulus were adjusted such that subjects' performance was $70 \%$ for that stimulus type. To ensure the overall luminance of the peripheral stimulus was consistent, the sum of these $\alpha$ parameters was always equal to 1 . For instance, in blocks 1 and 2 , SOA for the peripheral stimulus was initially fixed at $250 \mathrm{~ms}$. This meant the discrimination task was relatively easy so $\alpha$ levels tended to be low to achieve $70 \%$ performance threshold (for instance, $\boldsymbol{\alpha}_{\text {Face }} \cong 0.2$ and $\boldsymbol{\alpha}_{\text {Disk }} \cong 0.8$ in the single-peripheral-face task, $\boldsymbol{\alpha}_{\text {Face }} \cong 0.7$ and $\boldsymbol{\alpha}_{\text {Disk }} \cong 0.3$ in the single-peripheral-disk task).

In single-task blocks with SOA adjustment the $\alpha$ level for each aspect of the blended stimulus was computed by averaging between the threshold $\boldsymbol{\alpha}$ values from the previous $\alpha$ adjustment blocks. To ensure this averaging retained an overall $\alpha$ of 1 , mean $\boldsymbol{\alpha}$ for disk $\left(\boldsymbol{\alpha}_{\mathrm{mDisk}}\right)$ and face $\left(\boldsymbol{\alpha}_{\mathrm{mFace}}\right)$ were computed as follows. In blocks 3,4 , and 5 from the example outlined above, mean $\alpha$ levels would be:

$$
\begin{aligned}
& \alpha_{m D i s k}=\frac{\alpha_{\text {Disk }}+\left(1-\alpha_{\text {Face }}\right)}{2}=\frac{0.3+(1-0.2)}{2}=0.55 \\
& \alpha_{m D i s k}=\frac{\alpha_{\text {Face }}+\left(1-\alpha_{\text {Disk }}\right)}{2}=\frac{0.2+(1-0.3)}{2}=0.45
\end{aligned}
$$

SOA for the peripheral stimulus was then adjusted to achieve $70 \%$ performance accuracy for the relevant stimulus (for instance, in the example provided we would expect SOA to be shorter than $250 \mathrm{~ms}$ for both disks and faces). The mean SOA across peripheral conditions was then applied to the next $\alpha$ adjustment block and the staircasing procedure was repeated in blocks 6 through 10 .

This process meant we efficiently established SOAs and $\alpha$ values with which each subject performed the single-peripheral-face and single-peripheral-disk tasks at $70 \%$ accuracy. To complete training, subjects were presented with 20 trials of the dual-task peripheral-face + central-letter condition and 20 trials of the dual-task peripheral-disk + central letter condition. In this and subsequent dual-task blocks, mean 
SOA and mean $\alpha$ levels were applied from the preceding block. This ensured stimulus appearance was identical for both dual-task conditions.

\section{Data collection during the main testing runs}

Training and testing for Experiment 3 was conducted over 3 sessions on 3 separate days. In Session 1, after the training described above, we tested subjects in two runs of the main experiment. Each run included a 30 trial block of each of our 5 conditions: single-central-letter, single-peripheral-disk, single-peripheral-face, dual-letter-disk, and dual-letter-face tasks. We adjusted SOAs for the central letter task with QUEST in every single-central-letter block. In alternate runs of the single-peripheral blocks we adjusted the $\alpha$ level (even runs) or SOA (odd runs) for the peripheral stimuli. During the dual-task blocks, we used $\boldsymbol{\alpha}$ levels and SOAs that were updated in the preceding single-task blocks. Sessions two and three involved 3 runs of the main experiment each. Thus, excluding training, subjects completed 8 blocks for each of the five task conditions.

\section{Perceptual Awareness Scales}

As subjective ratings for Experiment 3, we employed Perceptual Awareness Scales (PAS) (Ramsøy \& Overgaard, 2004; Sandberg, Timmermans, Overgaard, \& Cleeremans, 2010), which is a more direct measure of conscious perception. For this purpose, we changed the display labels from "sure" and "not sure" into "easy to see" and "hard to see" and instructed subjects to rate ' 1 ' when the stimulus was very hard to see and ' 4 ' when the stimulus was very easy to see. We did not explicitly describe the ratings of ' 2 ' and ' 3 ' but we encouraged subjects to use all 4 levels when appropriate.

\section{Results and Discussion}

Face-aspect, but not colour-aspect, of the blended stimulus can be discriminated in the near absence of attention

When we merged a face and a disk through $\alpha$-blending in Experiment 3, subjects continued to possess discriminability for the face-, but not the disk-, aspect of the blended stimulus (Figure $3 \mathrm{G}$ and J, Table 7 ). When subjects paid close attention to the 
central letter task, maintaining objective performance (Type 1 AUC) at 0.81 , objective discrimination performance for the face-gender aspect of the blended stimulus remained above chance (0.71) while disk-colour discrimination fell to chance (0.53). Both stimulus aspects were performed well above chance $(\sim 0.75)$ in the respective single-task conditions. Two-way within-subject ANOVAs confirmed significant main effects of attention (single vs dual, $p<.001$ ), stimulus type (face vs disk, $p<.001$ ) and their interaction $(p<.001)$ for peripheral task performance. $T A^{\text {actual }}$ for face discrimination was 0.76 . In contrast, $\mathrm{TA}^{\text {actual }}$ for disk-colour was 0.08 , implying complete tradeoff in this feature.

Table 7. Objective Performance in Experiment 3

\begin{tabular}{|c|c|c|c|c|c|c|}
\hline \multirow[b]{2}{*}{ EXP 3} & \multirow[b]{2}{*}{ ST } & \multirow[b]{2}{*}{ face-DT } & \multirow[b]{2}{*}{ disk-DT } & \multicolumn{3}{|c|}{ Two-way ANOVA p-values } \\
\hline & & & & $\begin{array}{c}\text { stimulus } \\
\text { [face vs disk] }\end{array}$ & attention & $\begin{array}{l}\text { stimulus } \mathrm{x} \\
\text { attention }\end{array}$ \\
\hline Blended Faces & $.76 \pm .014$ & $.71 \pm .030$ & - & \multirow{2}{*}{$<.001$} & \multirow{2}{*}{$<.001$} & \multirow{2}{*}{$<.001$} \\
\hline Blended Disks & $.75 \pm .017$ & - & $.53 \pm .012$ & & & \\
\hline \multirow[t]{3}{*}{ Letter } & $.81 \pm .016$ & $.80 \pm .020$ & $.79 \pm .019$ & & & \\
\hline & \multicolumn{3}{|c|}{ TA $^{\text {actual }}$} & & & \\
\hline & mean \pm sem & $p$ against 1 & $p$ against 0 & & & \\
\hline $\begin{array}{c}\text { Letter + Blended } \\
\text { Faces }\end{array}$ & $.76 \pm .072$ & $<.001$ & 0.012 & & & \\
\hline $\begin{array}{c}\text { Letter + Blended } \\
\text { Disks }\end{array}$ & $.08 \pm .074$ & $>.25$ & $<.001$ & & & \\
\hline
\end{tabular}

\section{Perceptual awareness reduced for both features in the near absence of attention}

In Experiment 3 we employed a Perceptual Awareness Scale (PAS) to assess subjective experience (Ramsøy \& Overgaard, 2004). The relationship between attention and correctness on PAS ratings in Experiment 3 were examined using LME analyses for each stimulus type. For blended-disk discriminations, the full model with interaction 
term differed substantially from the reduced model $\left(\chi^{2}(1)=38.73, p<.001\right)$. In contrast, for blended-face discriminations a reduced model without interaction between attention and correctness did not differ from the full model $\left(\chi^{2}(1)=0.29, p>.25\right)$. The main effects of attention and correctness were highly significant when LME was applied to subset data (all $p<.001$ ). This confirmed that despite using the same stimuli and presentation parameters, the relationship between correctness and PAS was moderated by attention for disk-colour orientation but remained consistent between attention conditions when discriminating face-gender.

Table 8. PAS ratings in Experiment 3. We report the main effect of correctness (for each attention condition), attention (for correct and incorrect trials) and interaction in the same format as in Table $\mathbf{2}$ and 5.

\begin{tabular}{|c|c|c|c|c|}
\hline EXP 3 & ST & face-DT & disk-DT & \\
\hline Faces (correct) & $2.2 \pm .09$ & $2.0 \pm .12$ & - & $x^{2}=63.1, p<.001$ \\
\hline \multirow[t]{3}{*}{ Faces (incorrect) } & $1.9 \pm .12$ & $1.6 \pm .09$ & - & $x^{2}=50.5, p<.001$ \\
\hline & $x^{2}=74.6, p<.001$ & $x^{2}=108.1, p<.001$ & - & $\begin{array}{c}\text { Interaction: } \\
x^{2}=0.40, p>.25\end{array}$ \\
\hline & ST & face-DT & disk-DT & \\
\hline Disks (correct) & $2.2 \pm .19$ & - & $1.6 \pm .17$ & $x^{2}=326.9, p<.001$ \\
\hline \multirow[t]{3}{*}{ Disks (incorrect) } & $1.9 \pm .21$ & - & $1.5 \pm .17$ & $x^{2}=60.1, p<.001$ \\
\hline & $x^{2}=90.9, p<.001$ & - & $x^{2}=6.5, p=.01$ & $\begin{array}{c}\text { Interaction: } \\
\mathrm{x}^{2}=38.7, \mathrm{p}<.001\end{array}$ \\
\hline & ST & face-DT & disk-DT & \\
\hline Letter (correct) & $2.9 \pm .13$ & $2.7 \pm .17$ & $2.7 \pm .17$ & $x^{2}=47.7, p<.001$ \\
\hline \multirow[t]{2}{*}{ Letter (incorrect) } & $2.4 \pm .14$ & $2.3 \pm .13$ & $2.2 \pm .13$ & $x^{2}=18.0, p<.001$ \\
\hline & $x^{2}=105.6, p<.001$ & $x^{2}=95.5, p<.001$ & $x^{2}=141.4, p<.001$ & $\begin{array}{c}\text { Interaction: } \\
\mathrm{x}^{2}=.016, \mathrm{p}>.25\end{array}$ \\
\hline
\end{tabular}




\section{Metacognitive accuracy}

The results of metacognitive accuracy based on PAS in Experiment 3 were largely as expected from Experiment 1 and 2 with some exceptions (Figure $3 \mathrm{l}$ and L, Table 9). Under the dual-task, Type 2 AUC was high (0.59) for face-gender discrimination but near chance for disk-colour (0.53). Each was individually higher than chance $(\sim 0.58)$ in the single task condition. Subjects maintained similar levels of metacognition for the central letter task $(\sim 0.62)$ across all conditions. A two-way within-subject ANOVA on metacognitive accuracy for peripheral stimuli found a significant main effect of stimulus type ( $p=.032)$, but not for attention or their interaction (each $p>$.05). For the tradeoff analysis, we identified two outlier subjects (see below). After removal of the outliers, we confirmed that while metacognitive accuracy for the face-aspect remained intact $\left(T A^{\text {actual }}=0.90\right.$ (against $0, p=.031$; against $\left.1, p>.25\right)$ ), the disk-aspect dropped $(0.59, p>.05$ against both $1 \& 0)$.

Table 9. Metacognitive accuracy in Experiment 3

\begin{tabular}{|c|c|c|c|c|c|c|}
\hline \multirow[b]{2}{*}{ EXP 3} & \multirow[b]{2}{*}{ ST } & \multirow[b]{2}{*}{ face-DT } & \multirow[b]{2}{*}{ disk-DT } & \multicolumn{3}{|c|}{ Two-way ANOVA p-values } \\
\hline & & & & $\begin{array}{c}\text { stimulus } \\
\text { [disk vs face] }\end{array}$ & attention & $\begin{array}{l}\text { stimulus } \mathrm{x} \\
\text { attention }\end{array}$ \\
\hline Blended Faces & $.58 \pm .021$ & $.59 \pm .014$ & - & \multirow{2}{*}{0.032} & \multirow{2}{*}{0.232} & \multirow{2}{*}{0.082} \\
\hline Blended Disks & $.58 \pm .018$ & - & $.53 \pm .008$ & & & \\
\hline \multirow[t]{3}{*}{ Letter } & $.63 \pm .012$ & $.61 \pm .022$ & $.62 \pm .016$ & & & \\
\hline & \multicolumn{3}{|c|}{$\mathrm{TA}^{\text {actual }}$} & & & \\
\hline & mean \pm sem & $p$ against 0 & $p$ against 1 & & & \\
\hline Letters + Faces $(n=6)$ & $.91 \pm .320$ & 0.031 & $>.25$ & & & \\
\hline Letter + Disks $(n=8)$ & $.59 \pm .299$ & 0.091 & 0.208 & & & \\
\hline
\end{tabular}

Figure 5 lists the results of objective performance, PAS, and metacognitive accuracy for two subjects whose $\mathrm{TA}^{\text {actual }}$ values were greater than 3 standard deviations from the mean $T A^{\text {actual }}$ results for $n=32$ datasets pooled across Experiments 1-3. Figure 
$5 \mathrm{~B}$ and $5 \mathrm{E}$ show that the two subjects did not discriminate correct from incorrect trials under the single-task condition (i.e., overlapping red (correct) and faint red (incorrect) upright triangles along y-axis), which resulted in chance metacognitive accuracy for the single-task condition (upright triangles in Figure $5 \mathrm{C}$ and $\mathrm{F}$ ). Because these subjects were able to discriminate between correct and incorrect trials in the dual-task condition, our method for determining tradeoff resulted in $\mathrm{TA}^{\text {actual }}$ values that were massively positive ( 8.23 for Figure $5 \mathrm{C}$ ) and negative $(-7.96$ for Figure $5 \mathrm{~F})$. We will return to this issue in our General Discussion. 

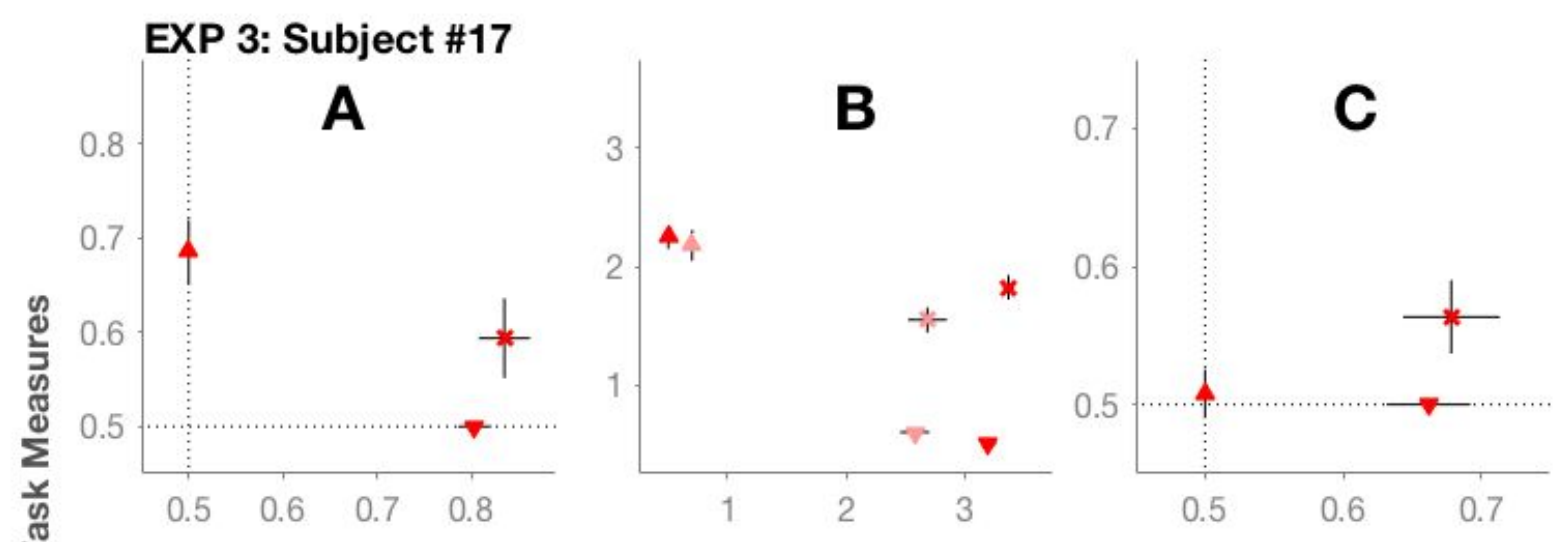

\section{EXP 3: Subject \#21}
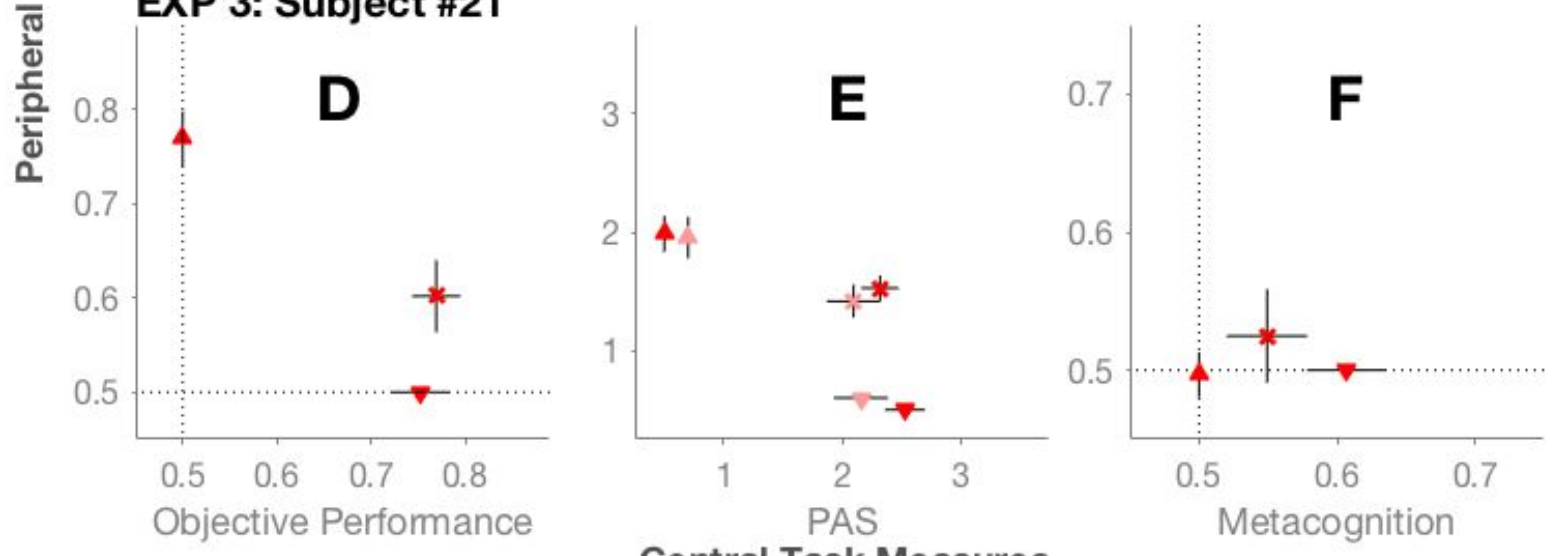

Central Task Measures

Figure 5. Two outlier subjects removed for tradeoff analysis of face-aspect metacognition in Experiment 3 (Table 9). Figure 5A-C for subject \#17, D-F for subject \#21. A and D for objective performance, $B$ and $E$ for PAS, C and F for metacognition. Note higher metacognitive accuracy for dual-task face discriminations than those in the single-task condition despite lower mean performance and perceptual awareness. Error bars reflect standard error of the mean over blocks. See Supplementary Figures $2 A-C$ for all subjects data in this format. 


\section{General Discussion}

In this paper, we addressed four criticisms of the dual-task paradigm: the issues of 1) metacognition, 2) saliency of faces, 3) excessive training, and 4) response interference. The most important of these was metacognition; whether successful discrimination of faces with little or no top-down attentional amplification is achieved with conscious access. We assessed this by computing metacognitive accuracy, the correspondence between subjects' accuracy and subjective reports (Fleming \& Lau, 2014; Galvin, Podd, Drga, \& Whitmore, 2003; Kaunitz et al., 2016; Kunimoto et al., 2001; Song et al., 2011). Metacognitive accuracy under the dual task has rarely been investigated (but see (Sherman, Seth, Barrett, \& Kanai, 2015; Stein \& Peelen, 2017)). Addressing conscious access for stimuli in the dual-task remains a critical limitation if this paradigm seeks to address the necessity debate (Jennings, 2015).

We assessed the correspondence between subjects' discrimination accuracy and subjective reports of either confidence (Experiment 1 and 2) or perceptual awareness (PAS, Experiment 3) using Type 2 AUC (Chen et al., 2016; Fleming \& Lau, 2014; Kaunitz et al., 2016; Kunimoto et al., 2001). For gender discrimination (Figure 3 A-F, Figure 4 A\&C, Table 1-3, 7-9), objective performance, confidence ratings, and metacognitive accuracy did not differ greatly between the single (i.e., attended) and dual (i.e., unattended) task conditions. When the same procedure was applied to the simple disk stimuli, performance collapsed in the near absence of attention (Figure $3 \mathrm{G}-\mathrm{L}$, Figure 4 B\&D, Table 4-9). Any evidence of above chance discrimination in this condition was accounted for by attentional tradeoff; subjects sacrificed performance on the central task in order to respond to disk stimuli.

This pattern of results was also found when we used perceptual awareness scales (PAS) (Figure 3 D-F, J-L, Figure 4 C\&D, Table 7-9). Subjects' PAS for gender discriminations was broadly unchanged in the dual-task and corresponded well with accuracy. This suggests that phenomenology of this perceptual feature (or simply, their appearance) remains largely unchanged when attention is diverted (see Figure $4 C$ ). We note that this result and the collapse in PAS for disk-colour in the near absence of 
attention is inconsistent with a study by (Rahnev et al., 2011). Using simple, grating stimuli they found that visibility ratings for orientation judgements were lower when subjects attended.

Our findings support the claim that certain stimulus categories, such as face-gender, remain consciously visible in the near absence of attention while other features, such as colour orientation, do not. However, accepting this conclusion hinges on our addressing the remaining criticisms of the dual-task: 2) the saliency of face stimuli, 3) excessive training, and 4) response interference.

It is possible that subjects' ability to distinguish gender in the periphery is a product of 2) the inherent salience of face stimuli; a category known to attract our attention (Cerf et al., 2009; Fletcher-Watson et al., 2008; Johnson et al., 1991). In Experiment 3, using an $\boldsymbol{\alpha}$-blended, face/disk stimulus, we examined whether gender and/or colour orientation could be discriminated in the dual-task. Critically, despite these stimuli being co-located and presented using equivalent SOAs in both conditions, face gender but not colour orientation could be successfully categorised when attention was diverted to the central task (Figure $3 \mathrm{D}-\mathrm{F}, \mathrm{J}-\mathrm{L}$, Figure $4 \mathrm{C}$ and D, Table 7-9). While diverting attention critically impairs subjects' ability to distinguish colour orientation, we conclude that face-gender remains discriminable not because faces attract attention but because this feature remains consciously accessible in the near absence of attention.

The third issue relates to 3 ) training in the dual-task paradigm, which in previous studies typically required subjects to complete thousands of trials before the main experiment began (F. F. Li et al., 2002; Reddy et al., 2006, 2004). Such extensive training may drastically alter neural circuits (Dux et al., 2009), affect consciousness (Merikle \& Joordens, 1997) and impact performance (Garner et al., 2014; Sigman \& Gilbert, 2000; Verghese et al., 2017). We employed a psychometric staircase procedure, in which criterion was reached in fewer than 100 trials; a reduction in excess of $97 \%$ when compared to traditional dual-task studies (F. F. Li et al., 2002; Reddy et al., 2006, 2004). We found that face-gender discrimination was still possible in the near absence of attention despite limited training (Exp 1 and 3). 
Reducing training potentially leaves room for subjects to improve their performance during the main experiment, but we did not observe such improvement. None of our ANOVA analyses found significant main effects of block or interaction between block and other factors in any experiments or measures. These findings demonstrate that face-gender discrimination under dual-task conditions is unlikely to result from perceptual learning during the training phase, but is an inherent capacity of the visual system or perceptual learning through life.

The final issue is 4) response-interference: could making a response on the central task result in subjects forgetting their answer for the peripheral disk task? Supporting this potential explanation, previous studies found some evidence that compared to complex stimuli, simple stimuli can be more effectively masked leaving shorter perceptual availability for subsequent reports (Houtkamp \& Braun, 2010; Nieuwenstein, Johnson, Kanai, \& Martens, 2007; Nieuwenstein \& Potter, 2006). Thus, in Experiment 2 we examined whether disk discriminations can be rescued if the reporting procedure is simplified. This was achieved using a partial-report paradigm to

reduce the load on perceptual memory. Our results (Figure 3D-F, Figure 4B, Table 4-6) clearly indicate that discrimination of colour orientation under the dual-task was not rescued even when the partial-report condition minimised the influence of response interference.

\section{Limitations of the study and future directions}

Using QUEST, we adjusted SOAs between discrimination targets and their masks in all experiments and the degree of stimulus transparency (i.e., $\alpha$ parameter) in Experiment 3, to equate task accuracy (or \% of correct) in the respective single-task conditions. This strategy is theoretically sound and compatible with previous dual-task studies (Lee et al., 1999; Pastukhov \& Braun, 2007) as long as we compare the objective task accuracy (Type 1 AUC) between the single and dual-task conditions.

However, it is not clear if the same logic can be applied to subjective ratings and metacognition (Type 2 AUC). In fact, we identified two outlier subjects (Figure 5) who 
clearly violate the assumptions of dual-task studies. These two subjects did not discriminate correct and incorrect trials in terms of PAS in the single-task but did so in the dual-task. There can be several possible reasons for these behaviours.

One possibility is that the instruction for rating perceptual awareness was not clear to subjects. Because we did not include examples of invisible and visible stimuli, it is possible that subjects were not sure when to assign PAS of 1 and 4 . Another possibility is that these outlier subjects used PAS "across" the single- and dual- task conditions, a phenomenon we call metacognitive saturation. Inspection of these subjects' Type 1 AUC and PAS reveals that their Type 1 AUC and PAS for the peripheral faces were higher for the single- than for the dual-task. When subjects apply a single criterion for subjective ratings across tasks of dissimilar difficulty, subjective ratings in the simple task can saturate (Fleming \& Lau, 2014; Peters, Ro, \& Lau, 2016).

This metacognitive saturation prevents Type 2 measures from adequately discriminating metacognitive sensitivity in the easy task and, by comparison, inflates the metacognitive accuracy of the difficult task despite subjective ratings being lower on average.

Both of these potential issues may also relate to genuine inter-individual differences in metacognition (Fleming, Weil, Nagy, Dolan, \& Rees, 2010; Song et al., 2011). In future studies, we may be able to reduce apparent individual differences by including stimuli that are clearly visible or invisible, thus setting clear reference stimuli for all participants. Further, we can take advantage of our expedited training procedure to test many more subjects and investigate true individual differences in metacognition.

\section{Conclusion}

By using subjective ratings and metacognitive accuracy, we showed that certain aspects (face-gender) of peripheral vision but not others (colour orientation) are consciously accessible in the near absence of top-down attention. This result was achieved despite minimal training and conforms with subjective reports and inferences from the dual-task literature in suggesting the phenomenological distinction of features such as face-gender might be independent from selective attention (Braun \& Julesz, 
1998; Koch \& Tsuchiya, 2007; F. F. Li et al., 2002; Reddy et al., 2004; Tsuchiya \& Koch, 2016; van Boxtel, 2017; van Boxtel et al., 2010). Using the methods we present here, future studies might explore a range of stimulus types and features to reveal many categories of conscious perception in the near absence of attention. In doing so we expect the distinction between top-down attention and consciousness might be clarified permitting a deeper understanding of the functional and neuronal properties of each phenomenon (Koch \& Tsuchiya, 2007; Tsuchiya \& Koch, 2016; van Boxtel et al., 2010).

\section{Acknowledgements}

We would like to thank Alex Robinson and Vanessa Corneille for helping the data collection and construction of Experiment 3. LK and NT were supported by the Australian Research Council Discovery Project (DP130100194, http://www.arc.gov.au/discovery-projects). LK was supported by a fellowship from the Japanese Society for the Promotion of Science (JSPS P15048). NT was supported by Precursory Research for Embryonic Science and Technology project from the JST (3630, http://www.jst.go.jp/kisoken/presto/en/about/) and Australian Research Council Future Fellowship (FT120100619, http://www.arc.gov.au/future-fellowships). 


\section{References}

Bates, D., Mächler, M., Bolker, B., \& Walker, S. (2015). Fitting Linear Mixed-Effects Models Usinglme4. Journal of Statistical Software, 67(1). https://doi.org/10.18637/jss.v067.i01

Bherer, L., Kramer, A. F., Peterson, M. S., Colcombe, S., Erickson, K., \& Becic, E. (2005). Training effects on dual-task performance: are there age-related differences in plasticity of attentional control? Psychology and Aging, 20(4), 695-709.

Bindemann, M., Burton, A. M., Hooge, I. T. C., Jenkins, R., \& de Haan, E. H. F. (2005). Faces retain attention. Psychonomic Bulletin \& Review, 12(6), 1048-1053.

Block, N. (2011). Perceptual consciousness overflows cognitive access. Trends in Cognitive Sciences, 15(12), 567-575.

Brainard, D. H. (1997). The Psychophysics Toolbox. Spatial Vision, 10(4), 433-436.

Braun, J. (1998). Vision and attention: the role of training. Nature, 393(6684), 424-425.

Braun, J., \& Julesz, B. (1998). Withdrawing attention at little or no cost: detection and discrimination tasks. Perception \& Psychophysics, 60(1), 1-23.

Braun, J., \& Sagi, D. (1990). Vision outside the focus of attention. Perception \& Psychophysics, 48(1), 45-58.

Cashon, C., \& Cohen, L. (2003). The construction, deconstruction, and reconstruction of infant face perception. In O. Pascalis \& A. Slater (Eds.), The development of face processing in infancy and early childhood: Current perspectives (pp. 55-68). New York: NOVA Science Publishers.

Cerf, M., Frady, E. P., \& Koch, C. (2009). Faces and text attract gaze independent of the task: Experimental data and computer model. Journal of Vision, 9(12), 10.1-15.

Chen, B., Mundy, M., \& Tsuchiya, N. (2016). Learning improves conscious access at the bottom, but not the top: Reverse hierarchical effects in perceptual learning and metacognition. https://doi.org/10.1101/073130

Cohen, M. A., Dennett, D. C., \& Kanwisher, N. (2016). What is the Bandwidth of Perceptual Experience? Trends in Cognitive Sciences, 20(5), 324-335.

Cousineau, D. (2005). Confidence intervals in within-subject designs: A simpler solution 
to Loftus and Masson's method. Tutorials in Quantitative Methods for Psychology, 1(1), 42-45.

Debner, J. A., \& Jacoby, L. L. (1994). Unconscious perception: attention, awareness, and control. Journal of Experimental Psychology. Learning, Memory, and Cognition, 20(2), 304-317.

Dehaene, S. (2014). Consciousness and the Brain. Singapore Books.

Dehaene, S., \& Changeux, J.-P. (2011). Experimental and theoretical approaches to conscious processing. Neuron, 70(2), 200-227.

Dux, P. E., Tombu, M. N., Harrison, S., Rogers, B. P., Tong, F., \& Marois, R. (2009). Training improves multitasking performance by increasing the speed of information processing in human prefrontal cortex. Neuron, 63(1), 127-138.

Fleming, S. M., \& Lau, H. C. (2014). How to measure metacognition. Frontiers in Human Neuroscience, 8, 443.

Fleming, S. M., Weil, R. S., Nagy, Z., Dolan, R. J., \& Rees, G. (2010). Relating introspective accuracy to individual differences in brain structure. Science, 329(5998), 1541-1543.

Fletcher-Watson, S., Findlay, J. M., Leekam, S. R., \& Benson, V. (2008). Rapid Detection of Person Information in a Naturalistic Scene. Perception, 37(4), 571-583.

Galvin, S. J., Podd, J. V., Drga, V., \& Whitmore, J. (2003). Type 2 tasks in the theory of signal detectability: discrimination between correct and incorrect decisions. Psychonomic Bulletin \& Review, 10(4), 843-876.

Garner, K. G., Tombu, M. N., \& Dux, P. E. (2014). The influence of training on the attentional blink and psychological refractory period. Attention, Perception \& Psychophysics, 76(4), 979-999.

Houtkamp, R., \& Braun, J. (2010). Cortical response to task-relevant stimuli presented outside the primary focus of attention. Journal of Cognitive Neuroscience, 22(9), 1980-1992.

Jennings, C. D. (2015). Consciousness Without Attention. Journal of the American 
Philosophical Association, 1(02), 276-295.

Jiang, Y., Costello, P., \& He, S. (2007). Processing of Invisible Stimuli: Advantage of Upright Faces and Recognizable Words in Overcoming Interocular Suppression. Psychological Science, 18(4), 349-355.

Jiang, Y., Yi, J., \& Sheng, H. (2006). Cortical Responses to Invisible Faces: Dissociating Subsystems for Facial-Information Processing. Current Biology: CB, 16(20), 2023-2029.

Johnson, M. H., Dziurawiec, S., Ellis, H., \& Morton, J. (1991). Newborns' preferential tracking of face-like stimuli and its subsequent decline. Cognition, 40(1-2), 1-19.

Joseph, J. S., Chun, M. M., \& Nakayama, K. (1997). Attentional requirements in a "preattentive" feature search task. Nature, 387(6635), 805-807.

Kaunitz, L. N., Rowe, E. G., \& Tsuchiya, N. (2016). Large Capacity of Conscious Access for Incidental Memories in Natural Scenes. Psychological Science. https://doi.org/10.1177/0956797616658869

Koch, C., \& Tsuchiya, N. (2007). Attention and consciousness: two distinct brain processes. Trends in Cognitive Sciences, 11(1), 16-22.

Kunimoto, C., Miller, J., \& Pashler, H. (2001). Confidence and accuracy of near-threshold discrimination responses. Consciousness and Cognition, 10(3), 294-340.

Lamme, V. A. F. (2010). What introspection has to offer, and where its limits lie. Cognitive Neuroscience, 1(3), 232-240.

Lee, D. K., Koch, C., \& Braun, J. (1999). Attentional capacity is undifferentiated: concurrent discrimination of form, color, and motion. Perception \& Psychophysics, 61(7), 1241-1255.

Li, F. F., VanRullen, R., Koch, C., \& Perona, P. (2002). Rapid natural scene categorization in the near absence of attention. Proceedings of the National Academy of Sciences of the United States of America, 99(14), 9596-9601.

Li, F.-F., van Rullen, R., Koch, C., \& Perona, P. (2005). Why does natural scene categorization require little attention? Exploring attentional requirements for natural 
and synthetic stimuli. Visual Cognition, 12(6), 893-924.

Mack, A., \& Rock, I. (1998). Inattentional Blindness. MIT Press.

Macmillan, N. A., \& Creelman, D. C. (2004). Detection Theory: A User's Guide. Psychology Press.

Merikle, P. M., \& Joordens, S. (1997). Parallels between Perception without Attention and Perception without Awareness. Consciousness and Cognition, 6(2/3), 219-236.

Nieuwenstein, M. R., Johnson, A., Kanai, R., \& Martens, S. (2007). Cross-task repetition amnesia: Impaired recall of RSVP targets held in memory for a secondary task. Acta Psychologica, 125(3), 319-333.

Nieuwenstein, M. R., \& Potter, M. C. (2006). Temporal limits of selection and memory encoding: A comparison of whole versus partial report in rapid serial visual presentation. Psychological Science, 17(6), 471-475.

O'Brien, F., \& Cousineau, D. (2014). Representing Error bars in within-subject designs in typical software packages. Tutorials in Quantitative Methods for Psychology, 10(1), 56-67.

Pastukhov, A., \& Braun, J. (2007). Perceptual reversals need no prompting by attention. Journal of Vision, 7(10), 5.1-17.

Persaud, N., Navindra, P., Peter, M., \& Alan, C. (2007). Post-decision wagering objectively measures awareness. Nature Neuroscience, 10(2), 257-261.

Peters, M. A. K., Ro, T., \& Lau, H. (2016). Who's afraid of response bias? Neuroscience of Consciousness, 2016(1), niw001.

Rahnev, D., Maniscalco, B., Graves, T., Huang, E., de Lange, F. P., \& Lau, H. (2011). Attention induces conservative subjective biases in visual perception. Nature Neuroscience, 14(12), 1513-1515.

Ramsøy, T. Z., \& Overgaard, M. (2004). Introspection and subliminal perception. Phenomenology and the Cognitive Sciences, 3(1), 1-23.

Reddy, L., Reddy, L., \& Koch, C. (2006). Face identification in the near-absence of focal attention. Vision Research, 46(15), 2336-2343. 
Reddy, L., Wilken, P., \& Koch, C. (2004). Face-gender discrimination is possible in the near-absence of attention. Journal of Vision, 4(2), 106-117.

Rosenthal, D. M. (2000). Consciousness, content, and metacognitive judgments. Consciousness and Cognition, 9(2 Pt 1), 203-214.

Sandberg, K., Timmermans, B., Overgaard, M., \& Cleeremans, A. (2010). Measuring consciousness: is one measure better than the other? Consciousness and Cognition, 19(4), 1069-1078.

Sherman, M. T., Seth, A. K., Barrett, A. B., \& Kanai, R. (2015). Prior expectations facilitate metacognition for perceptual decision. Consciousness and Cognition, 35, 53-65.

Sigman, M., \& Gilbert, C. D. (2000). Learning to find a shape. Nature Neuroscience, 3(3), 264-269.

Song, C., Kanai, R., Fleming, S. M., Weil, R. S., Schwarzkopf, D. S., \& Rees, G. (2011). Relating inter-individual differences in metacognitive performance on different perceptual tasks. Consciousness and Cognition, 20(4), 1787-1792.

Sperling, G. (1960). The Information Available in Brief Visual Presentations.

Sperling, G., \& Dosher, B. A. (1986). Strategy and optimization in human information processing. In K. R. Boff, L. Kaufman, \& J. P. Thomas (Eds.), Handbook of Perception and Human Performance (Vol. 1, pp. 1-65). New York: Wiley.

Stein, T., \& Peelen, M. V. (2017). Object detection in natural scenes: Independent effects of spatial and category-based attention. Attention, Perception \& Psychophysics, 79(3), 738-752.

Stoerig, P., \& Cowey, A. (2007). Blindsight. Current Biology: CB, 17(19), R822-4.

Theeuwes, J., \& Van der Stigchel, S. (2006). Faces capture attention: Evidence from inhibition of return. Visual Cognition, 13(6), 657-665.

Tsuchiya, N., \& Koch, C. (2016). The Relationship Between Consciousness and Top-Down Attention. In The Neurology of Conciousness (pp. 71-91).

van Boxtel, J. J. A. (2017). Different Signal Enhancement Pathways of Attention and Consciousness Underlie Perception in Humans. The Journal of Neuroscience: The 
Official Journal of the Society for Neuroscience, 37(24), 5912-5922.

van Boxtel, J. J. A., Tsuchiya, N., \& Koch, C. (2010). Consciousness and Attention: On Sufficiency and Necessity. Frontiers in Psychology, 1. https://doi.org/10.3389/fpsyg.2010.00217

VanRullen, R., Reddy, L., \& Koch, C. (2004). Visual search and dual tasks reveal two distinct attentional resources. Journal of Cognitive Neuroscience, 16(1), 4-14.

Verghese, A., Mattingley, J. B., Garner, K. G., \& Dux, P. E. (2017). Decision-Making Training Reduces the Attentional Blink. Journal of Experimental Psychology. Human Perception and Performance. https://doi.org/10.1037/xhp0000454

Watson, A. B., \& Pelli, D. G. (1983). Quest: A Bayesian adaptive psychometric method. Perception \& Psychophysics, 33(2), 113-120.

Weiskrantz, L., Warrington, E. K., Sanders, M. D., \& Marshall, J. (1974). Visual capacity in the hemianopic field following a restricted occipital ablation. Brain: A Journal of Neurology, 97(4), 709-728.

Wilimzig, C., Tsuchiya, N., Fahle, M., Einhäuser, W., \& Koch, C. (2008). Spatial attention increases performance but not subjective confidence in a discrimination task. Journal of Vision, 8(5), 7.

Wolfe, J. M. (1999). Inattentional amnesia. In V. Coltheart (Ed.), Fleeting Memories (pp. 71-94). MIT Press.

Wundt, W. (1874). Grundzüge der physiologischen Psychologie.

Yang, E., Brascamp, J., Kang, M.-S., \& Blake, R. (2014). On the use of continuous flash suppression for the study of visual processing outside of awareness. Frontiers in Psychology, 5. https://doi.org/10.3389/fpsyg.2014.00724 


\section{Supplementary Material}

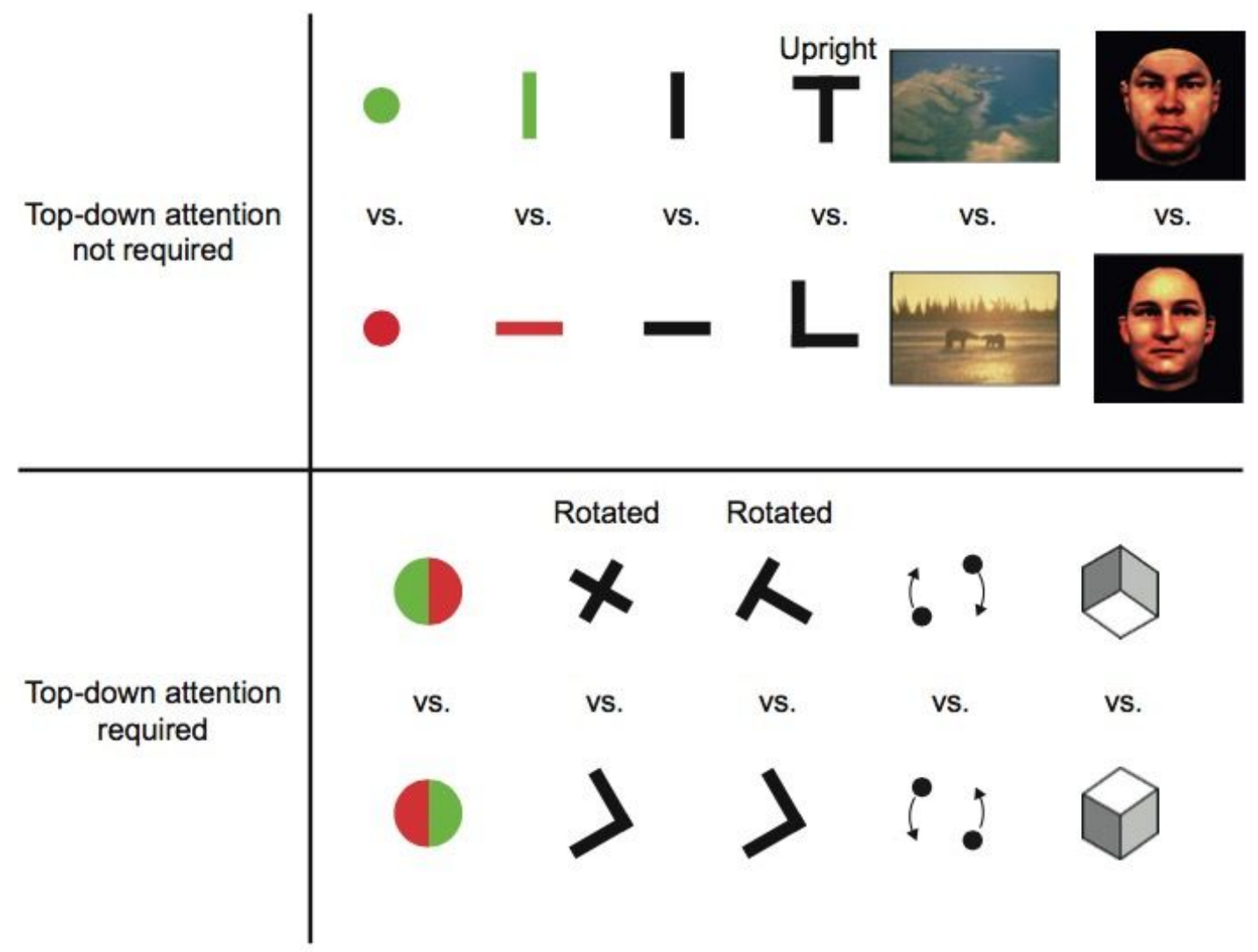

Supplementary Figure 1. Tsuchiya \& Koch (2015) 'The Relationship Between Consciousness and Top-Down Attention' 


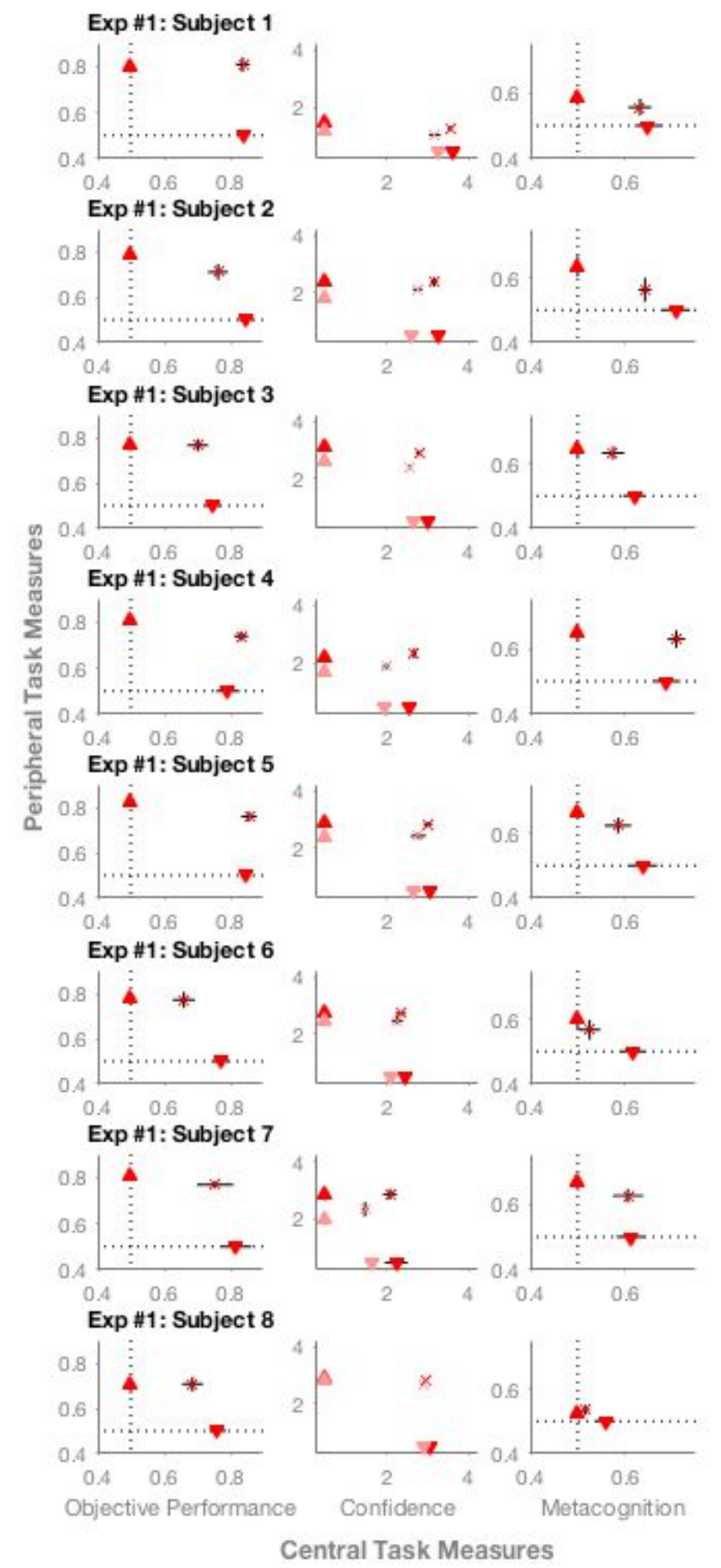

Supplementary Figure 2A. Individual subject data for Experiment 1 
Exp \#2: Subject 9
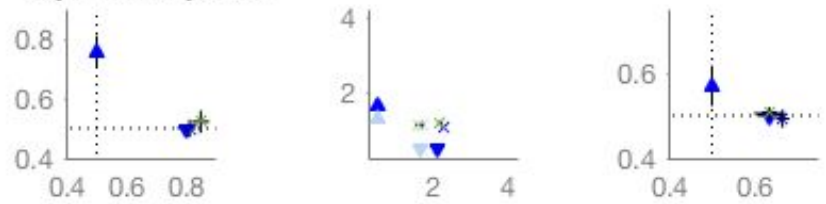

Exp \#2: Subject 10
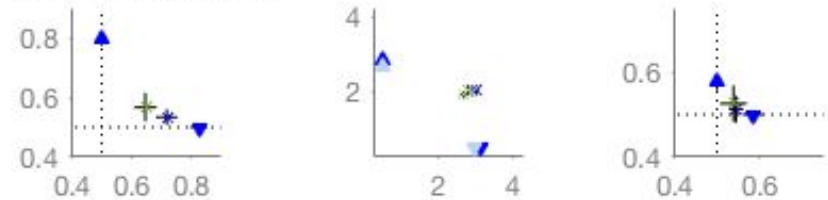

Exp \#2: Subject 11
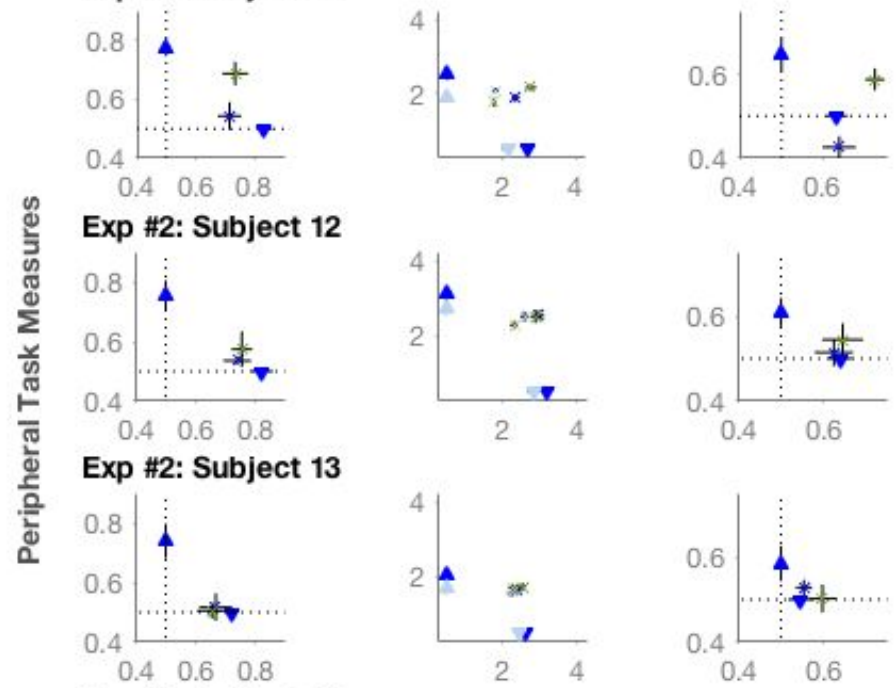

Exp \#2: Subject 14
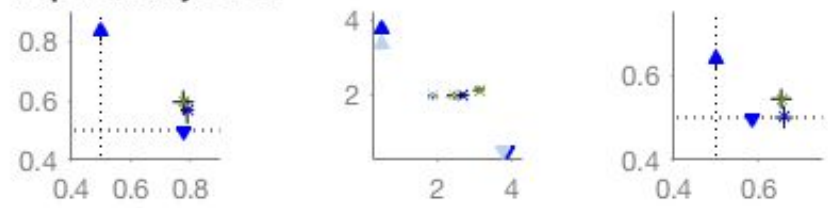

Exp \#2: Subject 15
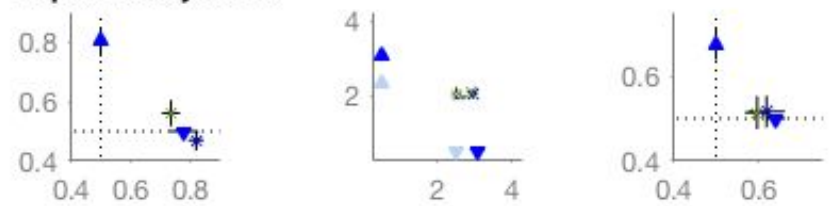

Exp \#2: Subject 16
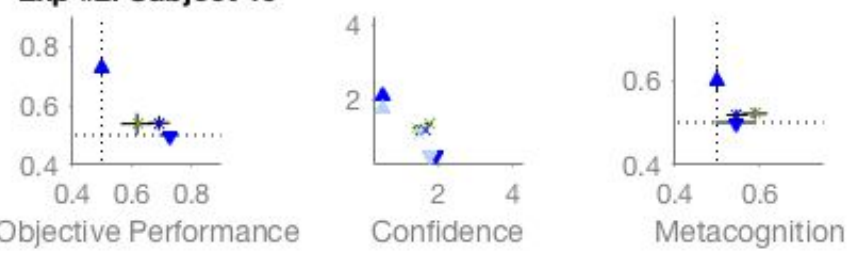

Central Task Measures

Supplementary Figure 2B. Individual subject data for Experiment 2 
FACES

DISKS

Exp \#3: Subject 17
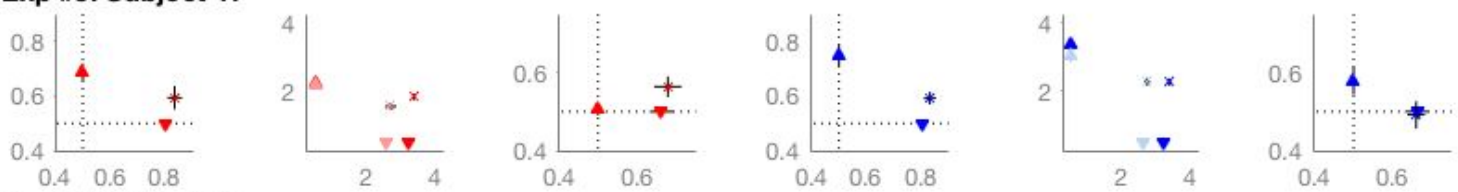

Exp \#3: Subject 18
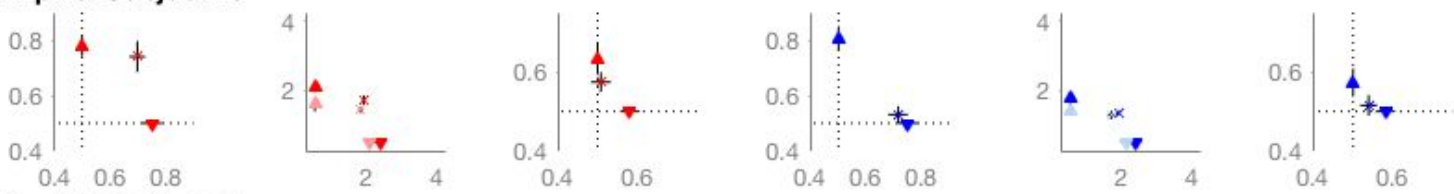

Exp \#3: Subject 19
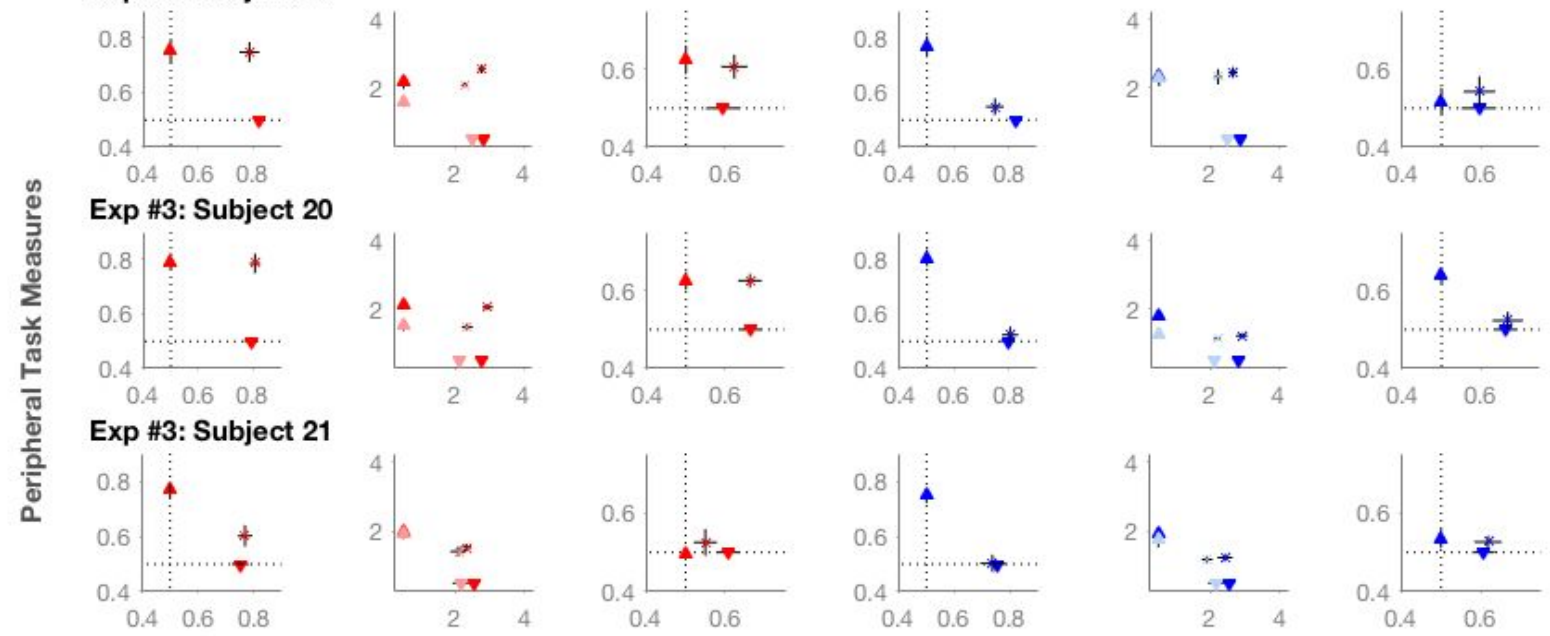

Exp \#3: Subject 22
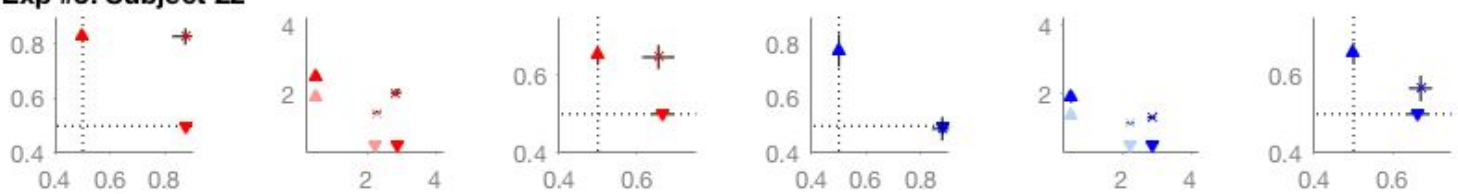

Exp \#3: Subject 23
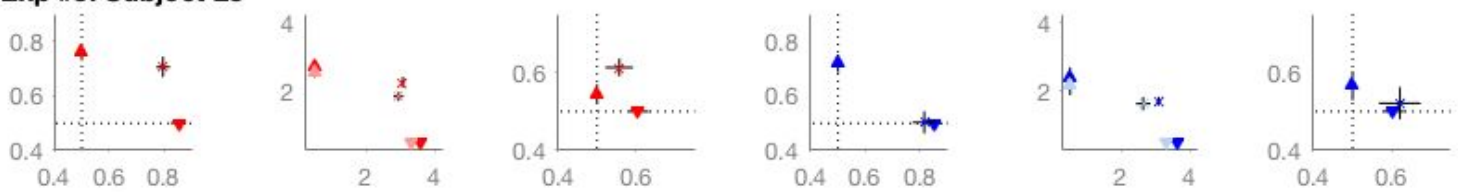

Exp \#3: Subject 24
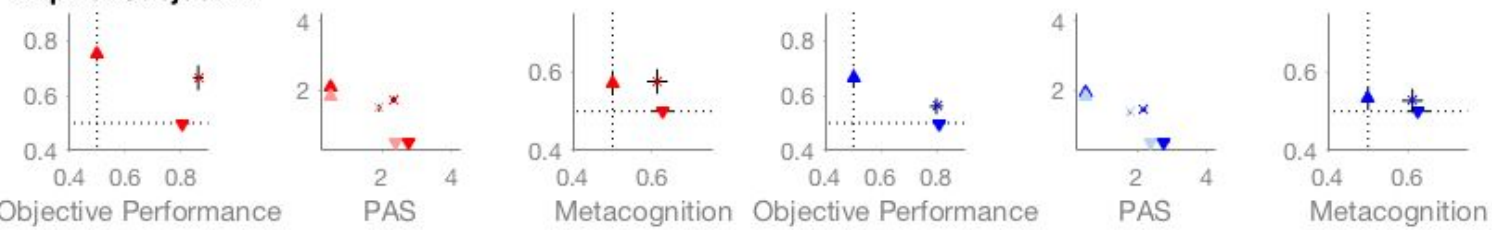

Central Task Measures

\section{Supplementary Figure 2C. Individual subject data for Experiment 3}

